ARTICLE

https://doi.org/10.1038/s41467-019-08520-7

\title{
Secreted metalloproteases ADAMTS9 and ADAMTS20 have a non-canonical role in ciliary vesicle growth during ciliogenesis
}

Sumeda Nandadasa ${ }^{1}$, Caroline M. Kraft', Lauren W. Wang ${ }^{1}$, Anna O'Donnell ${ }^{1}$, Rushabh Patel ${ }^{1}$, Heon Yung Gee $\mathbb{0}^{2}$, Kay Grobe $\mathbb{B}^{3}$, Timothy C. Cox ${ }^{4,5}$, Friedhelm Hildebrandt ${ }^{6}{ }^{6} \&$ Suneel S. Apte ${ }^{1}$

Although hundreds of cytosolic or transmembrane molecules form the primary cilium, few secreted molecules are known to contribute to ciliogenesis. Here, homologous secreted metalloproteases ADAMTS9 and ADAMTS20 are identified as ciliogenesis regulators that act intracellularly. Secreted and furin-processed ADAMTS9 bound heparan sulfate and was internalized by LRP1, LRP2 and clathrin-mediated endocytosis to be gathered in Rab11 vesicles with a unique periciliary localization defined by super-resolution microscopy. CRISPR-Cas 9 inactivation of ADAMTS9 impaired ciliogenesis in RPE-1 cells, which was restored by catalytically active ADAMTS9 or ADAMTS20 acting in trans, but not by their proteolytically inactive mutants. Their mutagenesis in mice impaired neural and yolk sac ciliogenesis, leading to morphogenetic anomalies resulting from impaired hedgehog signaling, which is transduced by primary cilia. In addition to their cognate extracellular proteolytic activity, ADAMTS9 and ADAMTS20 thus have an additional proteolytic role intracellularly, revealing an unexpected regulatory dimension in ciliogenesis.

\footnotetext{
${ }^{1}$ Department of Biomedical Engineering- ND20, Cleveland Clinic Lerner Research Institute, Cleveland, OH 44195, USA. ${ }^{2}$ Department of Pharmacology, Yonsei University College of Medicine, 50-1 Yonsei-ro, Seoul 03722, South Korea. ${ }^{3}$ Institute of Physiological Chemistry and Pathobiochemistry and Cells-inMotion Cluster of Excellence (EXC1003-CiM), University of Münster, 48149 Münster, Germany. ${ }^{4}$ Division of Craniofacial Medicine, Department of Pediatrics, University of Washington, 1959 NE Pacific St, Seattle, WA 98195, USA. ${ }^{5}$ Department of Oral and Craniofacial Sciences, UMKC School of Dentistry, 650 E 25th St, Kansas City, MO 64108, USA. ${ }^{6}$ Division of Nephrology, Boston Children's Hospital, Harvard Medical School, 300 Longwood Avenue, Boston, MA 02115, USA. Correspondence and requests for materials should be addressed to S.S.A. (email: aptes@ccf.org)
} 
$\mathrm{P}$ roteolysis is an irreversible posttranslational modification with profound biological impact. Secreted and cell-surface proteases proteolytically modify extracellular matrix (ECM) and other secreted molecules, and a few also act intracellularly 1,2 but none are known to participate in the biogenesis of cellular organelles. The primary cilium, which is solitary and sessile, compared to the motile cilia of ciliated epithelium, is a unique cellular organelle that is intimately associated with the extracellular environment and found in most post-mitotic cells ${ }^{3}$. It has a microtubule core and is enriched in several hundred other components ${ }^{4}$. The primary cilium extends by microtubule growth from a basal body, which is the post-mitotic maternal centrosome, to form a ciliary axoneme and it has a role in transduction of a variety of signals, including of morphogens such as hedgehogs 5 . Molecules localizing to the ciliary compartment and the peri-centrosomal vesicles are tightly regulated by the cell, trafficking via recycling endocytic or secretory vesicles and gaining privileged entry to the cilium via a proximal axoneme transition zone, a "gatekeeper" at the base of the cilium ${ }^{6}$. Transmembrane components such as smoothened are targeted to the ciliary membrane not only by lateral transport through plasma membrane but also via a complex mechanism utilizing cilium-bound recycling endocytic vesicles from the recycling endosome ${ }^{7,8}$. Diverse gene mutations result in primary cilium malformation or dysfunction, and the resulting clinical disorders, termed ciliopathies, affect the development of the kidney, heart, eye, brain, as well as metabolism ${ }^{9}$. Several signaling pathways additional to hedgehog signaling are mediated by the primary cilium, including TGF $\beta$ and PDGFR $\alpha^{10}$. Here we have uncovered the existence of a metalloproteinase-rich vesicular compartment at the base of the cilium, defining its origins and functional impact and providing role for proteolysis in biogenesis of the cilium.

Among a large family of secreted proteases having a characteristic domain structure, ADAMTS9 and ADAMTS20 are highly conserved orthologs of the nematode protease Gon-1, which is essential for gonadogenesis ${ }^{11,12}$. Adamts9-null embryos die at 7 days of gestation without undergoing gastrulation. In contrast, spontaneous Adamts $20^{\mathrm{Bt}}$ mutants survive and have defined a role for ADAMTS20 in remodeling of dermal ECM that regulates melanocyte colonization of $\operatorname{skin}^{13,14}$. Canine and human ADAMTS20 variants are associated with cleft palate, cleft lip and syndactyly ${ }^{15}$. Adamts $20^{\mathrm{Bt} / \mathrm{Bt}}$ mice have a low incidence of cleft palate, but upon deletion of one Adamts 9 allele, they have fully penetrant cleft palate and further reduction in pigmented hair follicles $^{16,17}$. ADAMTS9 and ADAMTS20 participate in regression of interdigital webs via cleavage of the proteoglycan versican, a major component of the embryonic $\mathrm{ECM}^{18}$ and versican accumulates in anomalies resulting from ADAMTS9 or ADAMTS20 inactivation ${ }^{16-18}$, identifying it as a key ADAMTS9 and ADAMTS20 substrate.

Here, we show that combined inactivation of both ADAMTS9 and ADAMTS20 impairs formation of primary cilia and causes severe developmental anomalies, which include craniofacial malformations and neural tube defects. These findings provide unexpected insights on ciliogenesis and a non-canonical intracellular role for proteases hitherto thought to have exclusively extracellular actions.

\section{Results}

ADAMTS9 and ADAMTS20 are enriched at the primary cilium base. ADAMTS9 and ADAMTS20 are secreted proteases known to proteolytically cleave the ECM component versican. Surprisingly, several new ADAMTS9- and ADAMTS20 monospecific polyclonal antibodies we generated (Supplementary Fig. $1 \mathrm{a}-\mathrm{c})$ as well as commercial antibodies showed intense staining of ADAMTS9 and ADAMTS20 at the base of the primary cilium in human RPE- 1 cells, mouse IMCD-3 cells, mouse NIH-3T3 cells, and primary human dermal fibroblasts (HDFs) upon induction of ciliogenesis by $24 \mathrm{~h}$ of serum starvation (Fig. 1a-h). ADAMTS9 localized to the cilium base in each cell type, and surrounded the $\gamma$-tubulin-stained basal body (Fig. 1d). ADAMTS20 antibodies similarly stained the base of the primary cilium of HDFs and NIH-3T3 cells respectively, but not RPE-1 cells, which do not express ADAMTS20 (Fig. 1e-g). Deconvolution super-resolution confocal microscopy (DSCM), with a defined resolution of $140 \mathrm{~nm}$, consistently resolved ADAMTS9 localization to multiple well-circumscribed vesicular structures forming rosette-like patterns at the base of primary cilia (Fig. 11, Supplementary Fig. 2a). Spatial mapping of ADAMTS9+ vesicles using DSCM revealed distinct vesicle populations, one comprising relatively small vesicles (average diameter $190 \mathrm{~nm}$ ) distributed extensively across the cell surface and not vicinal to the centrosome, whereas significantly larger (average diameter $296 \mathrm{~nm}$ ) vesicles were located in a barrel-shaped distribution $625.4 \pm 109.0$ $\mathrm{nm}$ from the centrosomal axis (Fig. 1j, k). To define their precise spatial relationship to the basal body and the cell membrane, we localized ADAMTS9 and centrosome appendage-specific markers by DSCM. ADAMTS9-stained vesicular rosettes were further lateral to and nearer the cell surface than the outermost boundary of the centrosome defined by CEP170, a sub-distal appendage (SDA) marker $^{19}$ (Fig. 1l, m). ADAMTS9 showed minimal overlap with the distal appendage marker CEP164 ${ }^{19}$, being located further lateral to it, but at a similar distance from the cell membrane (Fig. 1n). Immunogold electron microscopy revealed intracellular gold particles labeling ADAMTS9 that surrounded the basal body $(625.4 \pm 109.0 \mathrm{~nm}$ from its axis (Fig. 1o-q)) consistent with DSCM. The pre-embedding immunostaining method used precluded the observation of membranous vesicles due to the detergents used to permeabilize cells.

Secreted ADAMTS9 undergoes receptor-mediated endocytosis. An ADAMTS9 propeptide antibody gave no staining at the base of the cilium, despite recognition of ADAMTS9 zymogen on western blots (Supplementary Fig. 2b,c), indicating that ciliumassociated ADAMTS9 lacked the propeptide. Indeed, it was previously established that the ADAMTS9 propeptide was excised extracellularly by furin ${ }^{20}$, suggesting the interesting possibility that ADAMTS9-positive vesicle-like structures at the base of the cilium contained secreted, furin-processed and endocytosed ADAMTS9. Antibodies to clathrin heavy chain (CHC) or endocytosed Alexa 647 fluor-labeled transferrin mark early endocytic vesicles surrounding the cilium ${ }^{21,22}$ (Fig. 2a, b), but ADAMTS9 staining overlapped minimally with such vesicles. In contrast, costaining with Rab11, a post-recycling endocytic vesicle marker, showed considerable overlap with ADAMTS9 at the cilium base (Fig. 2c, d). Rab11+ and Rabin8+ recycling endocytic vesicles contribute to the ciliary vesicle ${ }^{23}$, whose growth at the distal end of the basal body, thus transporting cargo essential for the growing cilium, is a necessary process in ciliogenesis ${ }^{24-26}$. To test if ADAMTS9 localization to the cilium required endocytosis, we depleted CHC mRNA (Fig. 2e-h), which significantly reduced ADAMTS9+ vesicles at the cilium base (Fig. 2e, f, i, j), as well as ciliogenesis in RPE-1 cells (Fig. 2k). Inhibition of clathrinmediated endocytosis starting $24 \mathrm{~h}$ after serum starvation (and thus following cilium formation) using the clathrin inhibitors Dyngo4A or Pitstop2 eliminated ADAMTS9 localization at the cilium (Fig. 2l). Dyngo4A reduced cilium length, but neither inhibitor affected the percentage of ciliated cells (Supplementary Fig. 3b,c). This finding suggested that ADAMTS9 was essential for ciliogenesis, and likely to be necessary for maintenance of 

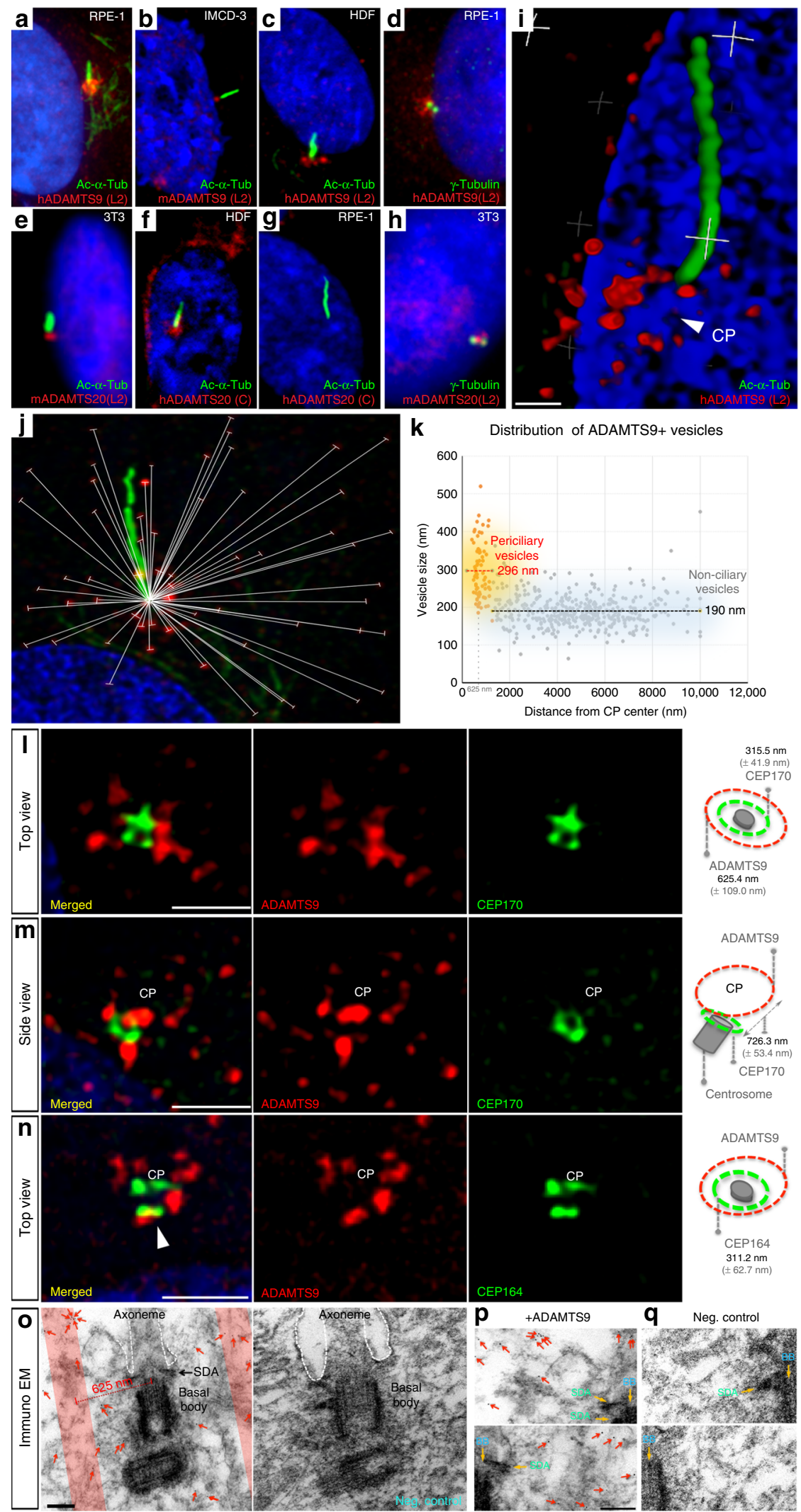

cilium length. Taken together, the results suggested that secreted ADAMTS9 was recycled, localizing to a subpopulation of late recycling endocytic vesicles targeted to the primary cilium in a highly dynamic process necessary for ciliogenesis.
A yeast-two hybrid screen for ADAMTS9 interactors using its Gon1-domain identified low-density lipoprotein receptor-related protein-1 (LRP1), which mediates receptor-mediated endocytosis of several metalloproteinases, including ADAMTS4 and 
Fig. 1 ADAMTS9 and ADAMTS20 localize to the cilium base. a-c Immunostaining for primary cilia (acetylated $\alpha$-tubulin, green), and human or mouse ADAMTS9 (red), shows ADAMTS9 localization at the primary cilium base in serum-starved RPE-1 cells (a), IMCD-3 cells (b), and human dermal fibroblasts (HDFs) (c). d Co-immunostaining of $\gamma$-tubulin (green) shows ADAMTS9 (red) around the basal body. e-g Focal ADAMTS20 staining (red) is present at the base of the primary cilium of NIH-3T3 cells (e), and HDFs ( $\mathbf{f}$ ), but not RPE-1 cells ( $\mathbf{g}$ ). $\mathbf{h}$ ADAMTS2O (red) is adjacent to the basal body ( $\gamma$-tubulin, green) of $\mathrm{NIH}-3 \mathrm{~T} 3$ cells. i 3D-projection of deconvolution super-resolution confocal microscopy (DSCM) of RPE-1 cells (imaged at $\times 1000$ magnification) shows vesicle-like ADAMTS9 staining (red) at the primary cilium base (CP, presumed ciliary pocket, white arrowhead). $\mathbf{j}$, $\mathbf{k}$ Representative DSCM image used for determining size and geographic distribution of ADAMTS9+ vesicles (j) and scatter plot (k) summarizing the analysis. I, $\mathbf{m}$ ADAMTS9+ vesicles (red) are located lateral and distal (above) to CEP170 (green), which defines the lateral-most boundary of sub-distal appendages ( $315.5 \mathrm{~nm} \pm 41.9 \mathrm{~nm}$ SD).

n ADAMTS9+ vesicles (red) are located lateral to the distal appendages (CEP164 (green)) ( $311.2 \mathrm{~nm} \pm 62.7 \mathrm{~nm}$ SD). The arrowhead shows apparent overlap that was nonoverlapping in 3D. Right-hand panels depict relative localizations and radial distances measured by DSCM. o Immuno-EM of ADAMTS9 in RPE1 cells shows $5 \mathrm{~nm}$ gold localization (arrowheads) in a barrel-shaped zone (pink shading) surrounding the basal body and $625 \mathrm{~nm} \pm 110 \mathrm{~nm}$ from its center. Secondary antibody-only negative control lacks gold particles. p, q High magnification images show $5 \mathrm{~nm}$ gold particles (red arrows) localized to the left (top image) or right side (bottom image) of the basal body in the ADAMTS9-stained TEM samples, whereas none are visible in the negative control. Yellow arrows point to the basal body and the sub-distal appendages. Scale bars $=500 \mathrm{~nm}$ in $\mathbf{i}, 2 \mu \mathrm{m}$ in $\mathbf{I}-\mathbf{n}$ and $200 \mathrm{~nm}$ in o-p

ADAMTS5 $^{27}$. Previous work demonstrated that LRP3 was essential for ciliogenesis in RPE-1 cells ${ }^{7}$ and LRP2 for neural tube morphogenesis and Shh signaling in the mouse retina ${ }^{28}$. LRP-1 and LRP-2 knockdown individually or in combination followed by serum starvation significantly reduced ADAMTS9+ vesicles at the cilium base (Fig. $2 \mathrm{~m}-\mathrm{p}$ ). These results suggested that LRP-1 and LRP-2 and clathrin-mediated endocytosis were necessary for ADAMTS9 recycling.

ADAMTS9 and ADAMTS20 act as proteases in ciliogenesis. RPE1 cells express ADAMTS9 but not ADAMTS20 (Fig. 1a, g). CRISPR/Cas-9 targeting of exon 2 of ADAMTS9 (Fig. 3a) generated sequence-verified RPE-1 clones with heterozygous (RB4) and homozygous (D12) frameshift indels (Fig. 3a) without offtarget mutations (Supplementary Fig. 3f). Loss of ADAMTS9 immunostaining and western blotting of mutant cells validated ADAMTS9 inactivation (Fig. 3b-d) (Supplementary Fig. 3g-j). Serum-starved RB4 and D12 cells each showed significantly reduced ciliogenesis (Fig. 3e, f; Supplementary Fig. 3j,k) and significantly shorter primary cilia (Fig. $3 \mathrm{~g}$ ), indicating that in the absence of ADAMTS20, RPE-1 cells were highly sensitive to reduced ADAMTS9. Overexpression of ADAMTS9 and ADAMTS20 in RB4 and D12 cells restored ciliogenesis, but importantly, their catalytically inactive mutants did not (Fig. 3e-g), (Supplementary Fig. 3j,k). The C-terminal myc tag of transfected wild type or catalytically inactive ADAMTS9 localized peri-centrosomally (Fig. 3h, i). This finding demonstrated not only that ADAMTS9 acted via the secretory pathway (i.e., was secreted and subsequently internalized), but argued against a putative alternatively spliced gene product that could regulate ciliogenesis by its targeting to the cytosol. The periciliary localization of the catalytically inactive form also eliminated the possibility that it failed to rescue ciliogenesis owing to defective trafficking to the cilium base. Most significantly, serum-free medium from myc-tagged ADAMTS9-expressing HEK293T cells rescued the ciliogenesis defect of D12 cells, and myc-tag staining was observed at the cilium base, supporting trafficking by endocytic recycling and action in-trans (Fig. $3 \mathrm{j}-\mathrm{m}$ ). Consistent with a requirement for catalytic activity, overexpression of the metalloprotease inhibitor TIMP3, which inhibits ADAMTS proteases ${ }^{29}$, suppressed ciliogenesis (Fig. 3n, o). Overexpression of TIMP3 did not impair ADAMTS9 trafficking to Rab11+ vesicles ruling out altered trafficking of ADAMTS9 by TIMP3 and supporting blockade of proteolytic activity (Fig. 3p).

ADAMTS9 knockout arrests specific aspects of ciliogenesis. Immunostaining for the centrosome components CEP170 and
CEP164 revealed that formation of the centrosome and its distal and SDAs was unaffected in RB4 and D12 cells (Fig. 4a). TEM of serum-starved wild-type and D12 cells showed that the majority of the mutant basal bodies lacked prominent docked ciliary vesicles $(\mathrm{CV})$ whereas a significant proportion had distal appendage bound vesicles (DAV) or small CVs unfused to the cell membrane (Fig. 4b, c). Axoneme elongation requires basal body maturation, which involves removal of the centrosome distal cap protein $\mathrm{CP} 110^{30}$ from the basal body, but not the daughter centrosome, essentially halving CP110 protein levels upon serum starvation $^{31}$. DSCM of ADAMTS9-null D12 cells showed they retained CP110-staining of both centrosomes, contrasting with wild-type cells, which had a single CP110-marked centrosome (Fig. 4d, e). Quantitative RT-PCR analysis shows significantly lower levels of CP110 mRNA but western blotting showed significantly higher CP110 levels upon serum starvation in D12 cells compared to wild-type cells (Fig. $4 \mathrm{f}-\mathrm{h}$ ). In contrast, the endosomal membrane trafficking molecules EHD1 and EHD3, which play crucial roles in the early steps of ciliogenesis and CP110 cap removal $^{32}$ were unaffected by the loss of ADAMTS9 (Fig. $4 \mathrm{f}-\mathrm{h}$ ). Super-resolution imaging of wild-type RPE-1 cells serum-starved for $6 \mathrm{~h}$ to visualize early steps in ciliogenesis demonstrated Rab11 + vesicles near CP110 capped basal bodies (Fig. 4i) in agreement with previous reports. Since CP110 and the contents of Rab11+ vesicles are in distinct cellular compartments, increased CP110 observed in the mutant cells is unlikely due to direct proteolytic activity of ADAMTS9, which is intra-vesicular. The few short cilia found in D12 cells formed a transition zone marked by tectonin-2 immunostaining (Fig. 4j). Although D12 cells had a higher percentage of phospho-histone $\mathrm{H} 3$ positive cells in serum-replete cultures, their proliferation was comparable to wild-type cells after starvation (Fig. 4k). Our findings thus define the point of ADAMTS9 impact as occurring during ciliogenesis and directly affecting basal body maturation and ciliary vesicle growth stages (Fig. $41, \mathrm{~m})^{33}$

Adamts9 and Adamts20 combined mutants resemble ciliopathies. To determine the role of ADAMTS9 and ADAMTS20 on ciliogenesis and the resulting functional impact on morphogenesis, we analyzed mouse mutants of these proteases. Because of early lethality of Adamts9-null mutants, we utilized a hypomorphic allele, Adamts 9 Gt, which survives to 15 days of gestation $(\mathrm{E} 15)^{17}$. In combination with the Adamts $20^{B t}$ allele we found a severe morphogenetic defect suggesting defective ciliogenesis. Specifically, Adamts $9 \mathrm{Gt} / \mathrm{Gt} ;$ Adamts $20^{\mathrm{Bt} / \mathrm{Bt}}$ embryos, which also survived until 15 days of gestation, had severe developmental anomalies resembling ciliopathy mutations in mice (Supplementary Movies 1-4), including occipital exencephaly, indicative 

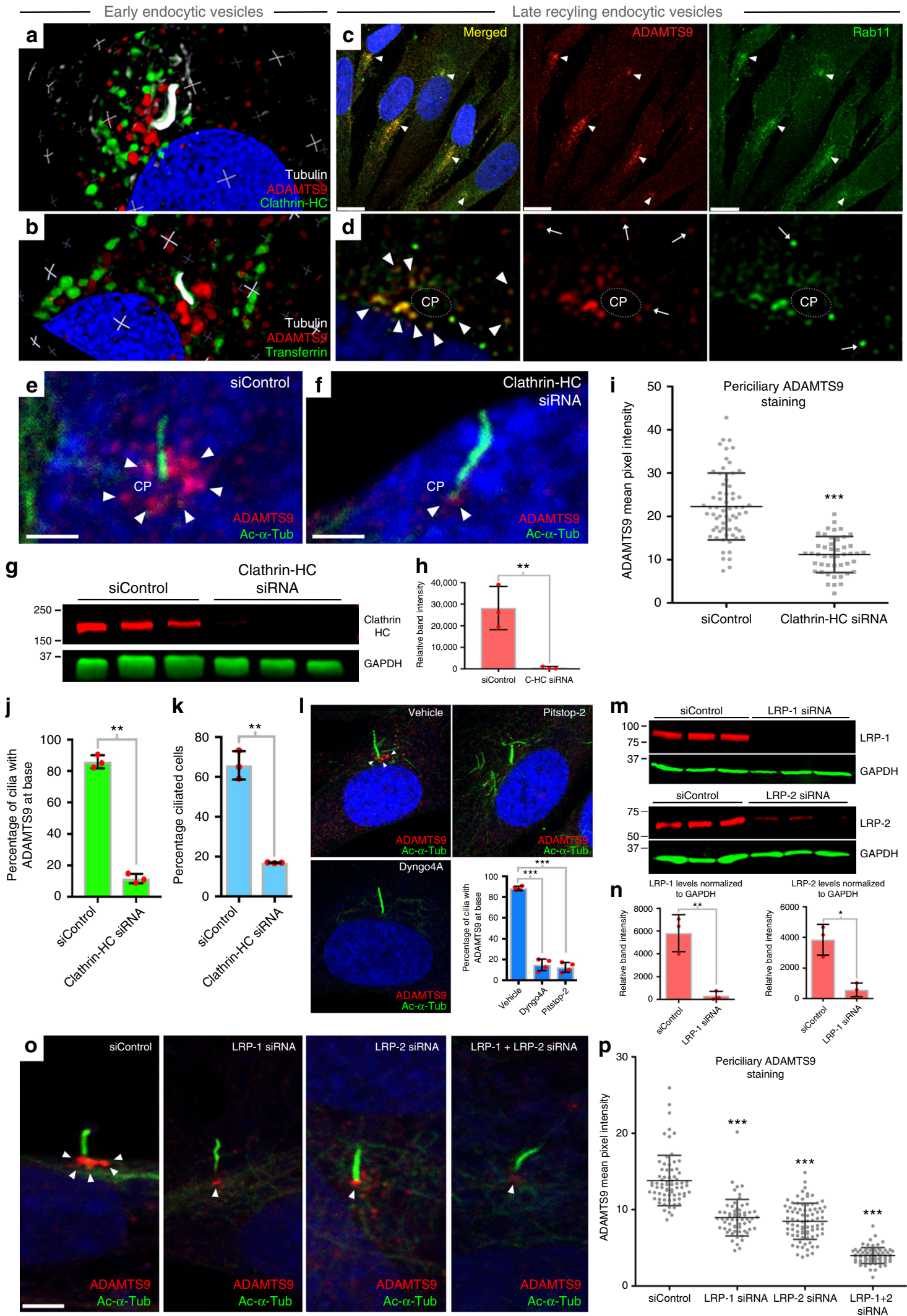

of their cooperative action in neural tube closure (Fig. 5a-c, Supplementary Fig. 4a-d). Other major developmental anomalies in these mutants included mandibular hypoplasia, eye defects, abnormal trachea-esophageal septation, increased facial width, cleft palate, cleft lip, and lateral asymmetry with the right-sided limbs abnormally close and the left-side limbs much further apart
(Supplementary Fig. 4e-h, Supplementary Movies 1-4). Body axis distortion led to visceral heterotaxy in mutant embryos (Supplementary Movies 3,4). Adamts 9 Gt/Gt embryos also had cleft palate (Supplementary Fig. 4i-l), and further Adamts 9 dosage reduction via combination of Adamts $9^{\mathrm{Gt}}$ with the inactivating Adamts $9^{\mathrm{Del}}$ allele (Adamts $9^{\mathrm{Gt} / \mathrm{Del}}$ ) resulted in failure to undergo 
Fig. 2 ADAMTS9 is internalized by clathrin-mediated endocytosis and transported to the periciliary region. $\mathbf{a}$, $\mathbf{b}$ 3D-projections of DSCM images of a ADAMTS9 (red), clathrin heavy chain (HC) (green), and tubulin (white), in RPE-1 cells show clathrin-coated endocytic vesicles surrounding the primary cilium but no overlap with ADAMTS9+ vesicles. b Endocytic vesicles (Alexa Fluor 647-transferrin, green) show minimal overlap with ADAMTS9+ vesicles at the cilium base. c, d Low magnification (c) and DSCM images (d) of ADAMTS9 (red) and Rab11 (green) show co-staining in RPE-1 cells. Arrowheads in d show co-stained vesicles surrounding the ciliary pocket (CP), and white arrows point to non-co-stained vesicles. e, $\mathbf{f}$ Clathrin-HC knockdown results in a loss of ADAMTS9 staining at the cilium base ( $N=3$ independent experiments). $\mathbf{g}$, $\mathbf{h}$ Western blot and clathrin-HC (red) quantification in RPE-1 cell lysates $48 \mathrm{~h}$ after siRNA transfection normalized to GAPDH (green) as control $\left(N=3\right.$ independent experiments, ${ }^{\star \star} p<0.001$, Student's $t$ test). i Quantification of ADAMTS9 pixel intensity at the cilium base shows significant reduction by clathrin-HC siRNA $\left(N=3\right.$ independent experiments, ${ }^{\star \star \star} p<0.0001$, Student's $t$ test). $\mathbf{j}$, $\mathbf{k}$ Percentage of ciliated cells and percentage of cilia with ADAMTS9 staining ( $N=3$ independent experiments, ${ }^{\star \star} p<0.001$; ${ }^{\star \star \star} p<0.0001$, Student's $t$ test). I Treatment of RPE-1 cells with the clathrin inhibitors Dyngo4A or Pitstop-2 after serum starvation for $24 \mathrm{~h}$ results in loss of ciliary ADAMTS9 staining $\left(N=4\right.$ independent experiments, ${ }^{\star \star \star} p<0.0001$, Student's $t$ test). $\mathbf{m}, \mathbf{n}$ Western blot and quantification of LRP-1 and LRP-2 $48 \mathrm{~h}$ after siRNA treatment shows depletion of LRP-1 and LRP-2 (normalized to GAPDH, green) ( $N=3$ independent experiments for each treatment, ${ }^{\star *} p<0.001$, ${ }^{*} p<0.05$, Student's $t$ test). - Immunostaining of RPE-1 cells with ADAMTS9 (red) and acetylated $\alpha$-tubulin (green), after serum starvation of LRP-1- and LRP-2-depleted cells or with combined depletion shows reduced ciliary ADAMTS9. p ADAMTS9 pixel intensity at the cilium base after treatment of LRP-1, LRP-2 or LRP-1+LRP2 siRNAs is significantly reduced compared to control siRNA levels $\left(N=3\right.$ independent experiments, ${ }^{\star \star \star} p<0.0001$, Student's $t$ test). Scale bars $=10 \mu m$ in c, $2 \mu \mathrm{m}$ in $\mathbf{e}, \mathbf{f}$ and $\mathbf{0}$. Bar charts and dotplots show mean and SD (error bars)

rotation, an open neural tube and death by E10.5 (Supplementary Fig. $4 \mathrm{~m}-\mathrm{p})$.

Intriguingly, Adamts 9 was strongly expressed in the midline neural floor plate from the onset of neural tube formation at E8.5 days until E11.5 but at lower levels in the lateral neural epithelium (Fig. 5d, Supplementary Fig. 5a). Adamts20 is expressed more laterally than Adamts 9 in the neural epithelium and at much lower levels in the ventral midline (Fig. 5e, Supplementary Fig. 5a). Adamts 9 and Adamts 20 mRNA overlapped with mRNA encoding their cognate substrate versican ${ }^{12}$, which was expressed strongly in the ventral midline embracing the Adamts9 domain, and in lateral neural epithelium (Fig. 5f) coinciding with Adamts20. The Adamts9 expression domain tightly coincided with the ventral floor plate Shh expression domain (Fig. 5g), but did not overlap with notochord Shh expression (Supplementary Fig. 5a). Along the length of the neural tube, Adamts 9 expression was limited to the floor plate in the hindbrain region and was absent further rostrally and caudally (Supplementary Fig. 5b), whereas Adamts20 showed extensive rostral-caudal expression ${ }^{14}$ and was expressed in the midline floor plate caudally (Supplementary Fig. 5a). The exclusive hindbrain exencephaly of Adamts ${ }^{G t} / G t$; Adamts $20^{B t / B t}$ mutants and Adamts $9^{G t / D e l}$ mutants further suggested a local role for ADAMTS9 alone as well as in partnership with ADAMTS20. Intriguingly, expression of Shh and Vcan were interdependent, i.e., Vcan hdf/hdf embryos (a null mutant ${ }^{34}$ ) had reduced Shh expression and Shh-null mice lacked Vcan mRNA in the neural tube epithelium (Supplementary Fig. 5c,d).

\footnotetext{
Adamts $9^{\mathrm{Gt} / \mathrm{G} t}$; Adamts20 ${ }^{B t / B t}$ embryos have ciliary defects. Confocal microscopy Z-stacks of thick vibratome sections coimmunostained for Smo and the ciliary axoneme marker IFT88 showed few primary cilia in Adamts ${ }^{G t / G t}$; Adamts $20^{B t / B t}$ neural tubes, whereas Smo localization to well-formed cilia was seen in the wild-type neural tube (Fig. 6a, Supplementary Fig. 6a, b). In wild type but not Adamts $9^{G t / G t ;}$ Adamts $20^{B t / B t}$ neural tubes Gli2-stained cilia were also marked by Arl13b (Fig. 6b, Supplementary Fig. 6c,d). Scanning electron microscopy (SEM) demonstrated rudimentary primary cilia in Adamts 9 Gt/Gt; Adamts $9^{B t / B t}$ and Adamts $9^{\mathrm{Gt} / \mathrm{Del}}$ neural tubes (Fig. 6c-e). A hallmark of mouse cilium mutants is impairment of Gli3 processing, which occurs in primary cilia ${ }^{35-38}$. Western blots showed more unprocessed Gli3 (Gli3-190), and less processed Gli3 repressor (Gli3-83) in Adamts $9 \mathrm{Gt} / \mathrm{Gt}$; Adamts20 ${ }^{\mathrm{Bt} / \mathrm{Bt}}$ heads (Fig. 6f, g). Signaling by all three Hh ligands, Shh, Ihh and Dhh relies on the primary cilium ${ }^{3}$. Upon Hh binding to Ptch1, repression of
}

Smoothened (Smo) is relieved and it relocates to the primary cilium to activate Gli transcription factors ${ }^{39}$. Reduced staining of Shh and the Shh receptor Patched-1, itself a Shh target, was seen in the Adamts $9^{\mathrm{Gt} / \mathrm{Gt}}$;Adamts $20^{\mathrm{Bt} / \mathrm{Bt}}$ rostral neural epithelium (Fig. 6h, i). The ventral neuronal markers activated by Shh signaling, Nkx6.1, Olig2, FOXA2 and Nkx2.2 were severely downregulated or aberrantly distributed in the mutant rostral neural tubes (Fig. 6j-m). Nkx6.1 and Olig2 staining of caudal neural tube sections, however, showed comparable staining in wild-type and mutant embryos indicating that impairment of Shh signaling was spatially limited to the rostral neural tube (Fig. 6n, o). RNA ISH analysis of Shh, Gli1, Gli2 and Ptch1 confirmed the downregulation of Shh responsive gene transcription in E9.5 mutant rostral neural tubes (Fig. 6p). Positional identity of neural epithelial cells is determined by counter-gradients of Shh (from the floor plate) and BMP/Wnt signaling (from the roof plate $)^{40,41}$. Exencephaly with reduced ventralization and ventral encroachment of dorsal neuronal markers is a characteristic finding in mouse cilium defects arising from mutated intraflagellar transport (IFT) proteins $s^{37}$, centrosome proteins ${ }^{42}$ and transition zone proteins ${ }^{43}$. Indeed, the dorsal neuronal markers Pax6 and Pax7 showed increased intensity and ventral expansion in the mutant neural tubes (Fig. 6q), accompanied by increased, ventrally expanded Bmp4 expression (Supplementary Fig. 6k,l), with normal expression of the BMP inhibitor Noggin (Supplementary Fig. $6 \mathrm{~m}, \mathrm{n})$. As in other exencephalic mutants Adamts $9 \mathrm{Gt} / \mathrm{Gt}$; Adamts $20^{\mathrm{Bt} / \mathrm{Bt}}$ neural tubes also showed increased cell proliferation (Fig. 6r). Since the observed neural epithelial cells of the mutants indeed do possess cilia, albeit short and bulbous, the observed ciliated cells are nonproliferating and thus excess proliferation is likely not causative in shortening of the cilium in the mutants. We therefore concluded that dysregulated Shh and BMP signaling as a result of reduction of primary cilia in the hindbrain was the likely mechanism of Adamts $9 \mathrm{Gt} / \mathrm{Gt}$; Adamts $20^{\mathrm{Bt} / \mathrm{Bt}}$ neural tube defects (Fig. 6s). Genetic interaction of Adamts9 with Ptch1 was tested and showed that Adamts $9^{\mathrm{Gt} / \mathrm{Gt}}$; Ptchl ${ }^{\text {lacZ/+ }}$ embryos consistently (3/3) developed occipital exencephaly, lateral asymmetry and abnormally positioned limbs similar to Adamts $9 \mathrm{Gt} / \mathrm{Gt}$; Adamts $20^{\mathrm{Bt} / \mathrm{Bt}}$ mutants (Supplementary Fig. $6 \mathrm{e}-\mathrm{g}$ ). Adamts $20^{\mathrm{Bt} /}$ Bt; Ptch $1^{\text {lacZl+ }}$ newborns (11/11) had fully penetrant cleft palate (Supplementary Fig. 6h,i), a phenotype previously observed in $100 \%$ of Adamts $20^{\mathrm{Bt} / \mathrm{Bt} ;}$ Adamts $9 \mathrm{Gt} /+$ mutants and $3 \%$ of Adamts $20^{\mathrm{Bt} / \mathrm{Bt}}$ mice ${ }^{16,17}$. Furthermore, Adamts $9^{\mathrm{Gt} /+}$; Ptch $1^{\mathrm{lacZ} /+}$ mice had highly penetrant hind limb preaxial polydactyly $(72 \%$, 8/11) (Supplementary Fig. 6j), a phenotype observed in only $2 \%$ of Ptch $1^{\text {lac } Z /+}$ mice ${ }^{44}$. Both Adamts 9 and Adamts 20 are expressed 
a

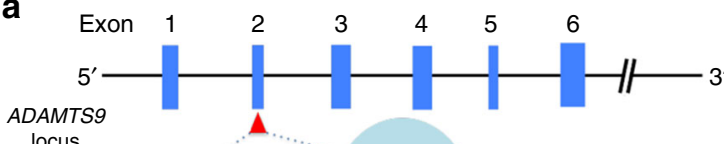

locus

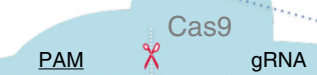

CACGAACGTCCACTTCAAAAGAACGCGACGGAGC GTGCTTGCAGGTGAAGTTTTCTTGCGCTGCCTCG

$\underline{R B 4}$

CACGA----------CAAAAGAACGCGACGGAGC

+/- CACGAACGTCCACTTCAAAAGAACGCGACGGAGC

D12 -1- CACGA--------CAAAAGAACGCGACGGAGC b

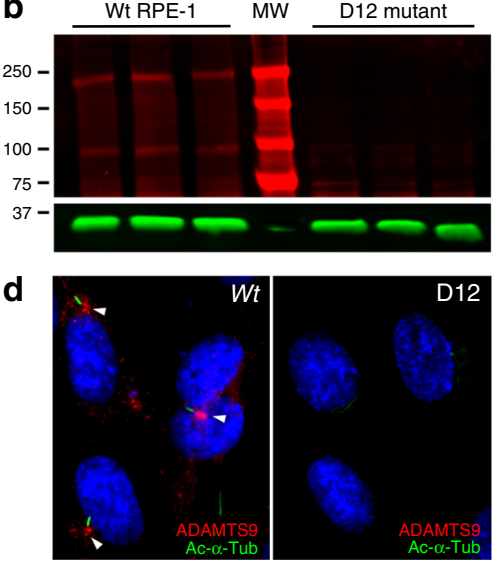

C

ADAMTS9 $(220 \mathrm{kDa})$ normalized to GAPDH

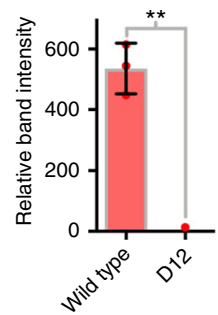

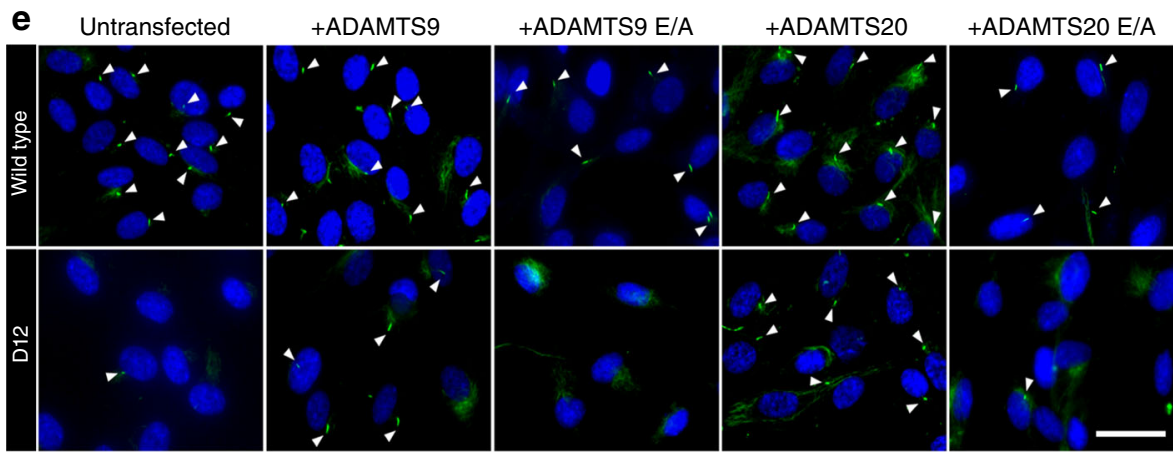
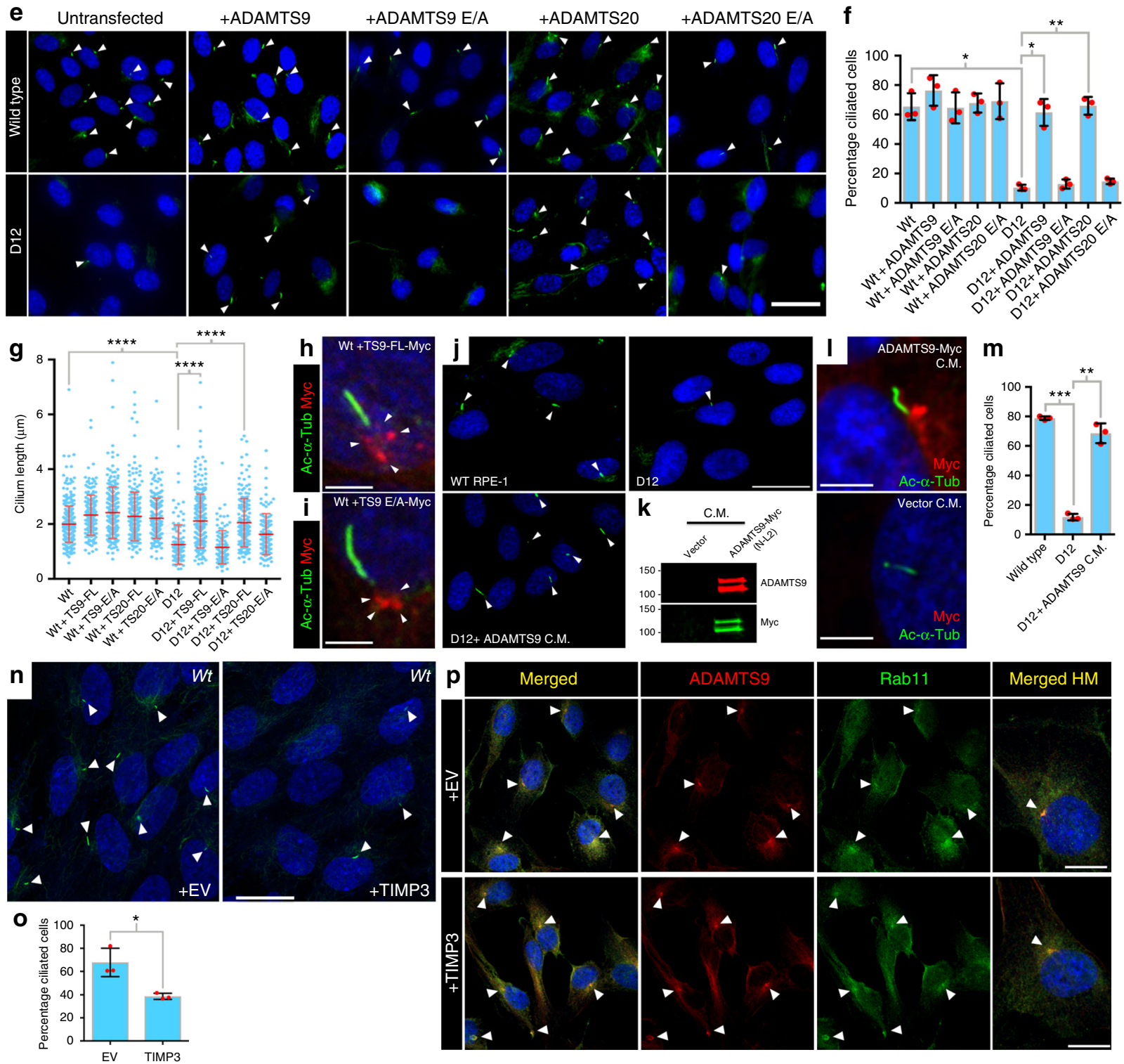

in the developing limb buds and are crucial for web regression $^{18,45,46}$ but polydactyly was seen neither after conditional deletion of Adamts945, nor in Adamts $9^{\mathrm{Gt}} / \mathrm{Gt} ;$ Adamts $20^{\mathrm{Bt} / \mathrm{Bt}}$ embryos.

ECM alterations in Adamts9 Att/Gt; Adamts20 $^{\mathrm{Bt} / \mathrm{Bt}}$ neural tubes. Since the cognate function of ADAMTS9 and ADAMTS20 is
ECM remodeling, we examined if loss of ADAMTS9 and ADAMTS20 affected ECM dynamics of the mouse neural epithelium, in addition to their role in ciliogenesis. Versican, an ADAMTS9 and ADAMTS20 substrate, is a large secreted chondroitin sulfate (CS) proteoglycan ${ }^{47,48}$. Intense CS staining and increased versican staining were seen in the Adamts9Gt/Gt; Adamts20 $0^{\mathrm{Bt} / \mathrm{Bt}}$ neural tube at E9.5 (Fig. 7a, b, Supplemental 
Fig. 3 ADAMTS9 and ADAMTS20 catalytic activity is essential and functionally redundant in ciliogenesis. a CRISPR-Cas9 inactivation of ADAMTS9 in RPE1 cells and sense-strand sequence of specific indels obtained in hemizygous (RB4) and homozygous (D12) RPE-1 clones. The PAM site and guide RNA (gRNA) sequences are shown in red and blue letters, respectively. b-d Western blotting (b, c) and immunofluorescence (d) shows elimination of ADAMTS9 in D12 cells. Arrowheads point to ADAMTS9 rich foci at the base of cilia. For (c), $N=3$ lanes each group, ${ }^{\star \star} p<0.001$. e- $\mathbf{g}$ Immunofluorescence of wild-type RPE-1 and D12 cells with acetylated $\alpha$-tubulin (green) after transfection with the indicated constructs (e) and the percentage of ciliated cells $(\mathbf{f})$ and cilium length $(\mathbf{g})$ resulting. Note presence of the primary cilium in D12 cells transfected with either wild-type ADAMTS9 or ADAMTS20, but not in the corresponding catalytically inactive (E/A) mutants $\left(N=3\right.$ independent experiments, ${ }^{\star \star \star *} p<0.00001 ;{ }^{* \star} p<0.001 ;{ }^{*} p<0.05$, Student's $t$ test). Arrowheads point to primary cilia. $\mathbf{h}$, i Transfected myc-tagged (red) ADAMTS9 (h) and ADAMTS9-E/A (i) localize to the base of the primary cilium (green) in wild-type RPE-1 cells. Arrowheads point to vesicles at the base of the primary cilium. $\mathbf{j}$ ADAMTS9-containing conditioned medium (C.M.) restores ciliogenesis in D12 cells. Arrowheads point to primary cilia. $\mathbf{k}$ Western blot of supplemented medium with anti-myc (green) or antiADAMTS9 (red). I Myc-tag (red) immunostaining shows concentration of exogenously supplied ADAMTS9 at the base of the cilium. $\mathbf{m}$ Quantitation of the percentage of ciliated cells in wild-type, D12 and D12 cells treated with exogenous ADAMTS9 shows rescue of ciliogenesis in D12 cells $(N=3$ independent experiments, ${ }^{\star \star \star} p<0.0001,{ }^{\star \star} p<0.001$, Student's $t$ test). $\mathbf{n}$, o Transfection of the metalloprotease inhibitor TIMP3 significantly reduces the percentage of ciliated RPE-1 cells ( $N=3$ independent experiments, ${ }^{*} p<0.05$, Student's $t$ test). $\mathbf{p}$ Transfection of the metalloprotease inhibitor TIMP3 does not affect ADAMTS9 (red) and Rab11 (green) colocalization. Arrowheads point to ADAMTS9/Rab11 rich foci in cells. Scale bars $=25 \mu \mathrm{m}$ in $\mathbf{e}, 20 \mu \mathrm{m}$ in $\mathbf{j}, \mathbf{n}$, $10 \mu \mathrm{m}$ in $\mathbf{p}, 3 \mu \mathrm{m}$ in h, i, I. Bar charts and dotplots show mean and S.D (error bars)

movie 5,6) and versican proteolysis revealed by the neo-epitope antibody anti-DPEAAE was reduced in the floor plate ${ }^{49}$ (Fig. $7 \mathrm{c}$ ). Versican binds to the glycosaminoglycan hyaluronan (HA) ${ }^{50}$ and Has2, the major HA synthase, is a direct target of the Shh signal transducers Gli1 and Gli2 ${ }^{51}$. In wild-type neural tubes HA was concentrated in the floor plate, a distribution perturbed in Adamts $9 \mathrm{Gt} / \mathrm{Gt} ;$ Adamts $20^{\mathrm{Bt} / \mathrm{Bt}}$ neural tubes (Fig. 7d). Increased HS-staining was seen in the mutant neural epithelium and surrounding mesenchyme (Fig. 7e). Consistent with a potential role in HS-proteoglycan turnover suggested by this finding, we observed direct ADAMTS9 binding to heparin and embryoderived heparan sulfate (Supplementary Fig. 6o,p). Fibronectin, a recently identified ADAMTS9 substrate (Wang et al., submitted) also stained more strongly in the mutant neural tube basement membrane and mesenchyme (Fig. 7f), whereas basement membrane molecules collagen IV, perlecan, and laminin were unaffected (Fig. 7d, g). Thus, proteoglycan-hyaluronan distribution in the neural tube was selectively affected by loss of Adamts 9 and Adamts20. Has2 mRNA expression was lost in the mutant neural tube floor plate as expected of an Shh target ${ }^{51}$, whereas Vcan and Fn1 mRNA were unaffected (Fig. 7h-j).

\begin{abstract}
Abnormal ciliary signaling and ECM in Adamts9 ${ }^{\mathrm{Gt}} / \mathrm{Gt}$ yolk sac. The primary cilium mediates Ihh signaling during yolk sac angiogenesis $^{52}$. Like $I h h^{-1-}$ embryos, Adamts ${ }^{G t / G t}$ embryos had poorly developed yolk sac microvasculature (Fig. 8a, b). Adamts 9 was specifically expressed in yolk sac mesoderm, including the mesothelium (Fig. 8c), whereas Adamts20 mRNA was absent in the yolk sac (Fig. 8d). Confocal and SEM imaging showed shorter primary cilia in Adamts $9^{G t / G t}$ yolk sac mesothelium (Fig. $8 \mathrm{e}-\mathrm{g}$ ). In-situ hybridization showed that $\mathrm{Hh}$ responsive mRNAs Gli1, Gli2, and Ptch1, expressed by mesoderm cells in wild-type yolk sac, were absent in Adamts $9^{G t / G t}$ yolk sacs (Fig. 8h). Adamts $9^{G t / G t}$ yolk sacs had more intense versican, fibronectin and hyaluronan staining and reduced versican cleavage, whereas collagen IV staining was unaltered (Fig. 8i). Consistent with ECM accumulation in the mutants, yolk sac mesoderm was thicker owing to an excess of amorphous, i.e., nonfibrillar ECM (Fig. 8j, k). RT-qPCR analysis of Vcan, Has2 and Fn1 was comparable, suggesting that versican and fibronectin accumulation ensued from reduced proteolytic turnover (Fig. 81). Together, the findings from Adamts $9 \mathrm{Gt} / \mathrm{Gt}$; Adamts $20^{\mathrm{Bt} / \mathrm{Bt}}$ and Adamts9Gt/Del neural tube and Adamts $9^{\mathrm{Gt} / \mathrm{Gt}}$ yolk sac indicate a dual role for ADAMTS9 and ADAMTS20, i.e., in ciliogenesis as well as ECM turnover.
\end{abstract}

Cilium defects are independent of versican proteolysis. We tested if the ADAMTS9-null RPE-1 cells had similar impairment of Shh responsiveness and versican remodeling and if the latter might have a role in perturbing ciliogenesis. Serum-starved wildtype and D12 cells were treated with the $500 \mathrm{~nm}$ smoothened agonist (SAG) for $12 \mathrm{~h}$ to induce Shh signaling. Staining with an SMO antibody and acetylated $\alpha$-tubulin showed ciliary axoneme localized SMO staining in wild-type cells and a lack of axoneme staining in D12 cells (Fig. 9a). Treatment with SAG neither rescued the percentage of ciliated cells nor their length in D12 cells (Fig. 9b, c). Quantitative RT-PCR revealed significantly low levels of Shh responsive (Ptch1, Gli1) gene expression in D12 cells in response to SAG treatment, although $S m o$, which is not a known Shh target, was also unexpectedly downregulated (Fig. 9d).

RPE-1 cells had low levels of Vcan mRNA expression and correspondingly, versican immunostaining, even in ADAMTS9 knockout D12 cells, during the 2-day culture period utilized for ciliogenesis assays (Supplementary Fig. 7a,b). However, upon culturing wild-type and D12 cells for 5 days we did observe versican accumulation in D12 cells and greater versican cleavage in wild-type cells (Supplementary Fig. 7c). Therefore, to test if increased pericellular versican could affect ciliogenesis, we overexpressed versican in RPE-1 cells (Fig. 9e, f). Despite the high level of versican resulting in their pericellular matrix, neither the percentage of ciliated cells nor cilium length were affected (Fig. 9g, h). Similarly, culturing either wild-type or D12 cells upon $20 \mu \mathrm{g} / \mathrm{mL}$ fibronectin-coated wells had no impact on ciliogenesis of wild-type cells nor did it rescue ciliogenesis in the D12 cells (Fig. 9i, j) (Supplementary Fig. 7d). Transfection of D12 cells with ADAMTS1 and ADAMTS5, which also cleave versican, did not restore ciliogenesis to wild-type levels, further supporting the conclusion that impaired processing of versican was not causally relevant to reduced ciliogenesis in the absence of ADAMTS9 (Fig. 9k). Previously genetic inactivation of ADAMTS16, a phylogenetically distantly related protease in rats, was associated with increased cilium length in renal tubular epithelium ${ }^{53}$. However, overexpression of Adamts16 in D12 cells did not rescue the ciliogenesis defect, although it did increase cilium length in wild-type cells. Conversely, knockdown of ADAMTS16 had no impact on ciliogenesis in wild-type or D12 cells (Fig. 91).

\section{Discussion}

Here, we show that ADAMTS9 working alone, or in partnership with ADAMTS20 where they are coexpressed is essential for ECM turnover and ciliogenesis during mammalian morphogenesis (Fig. 10a). The severe impact of their deficiency on cilium biogenesis provides an obvious mechanism for the observed impairment of Shh and Ihh signaling in the neural tube and yolk sac respectively. The findings also clearly illustrate a tissuespecific threshold for ADAMTS9 in ciliogenesis, i.e., in the yolk 

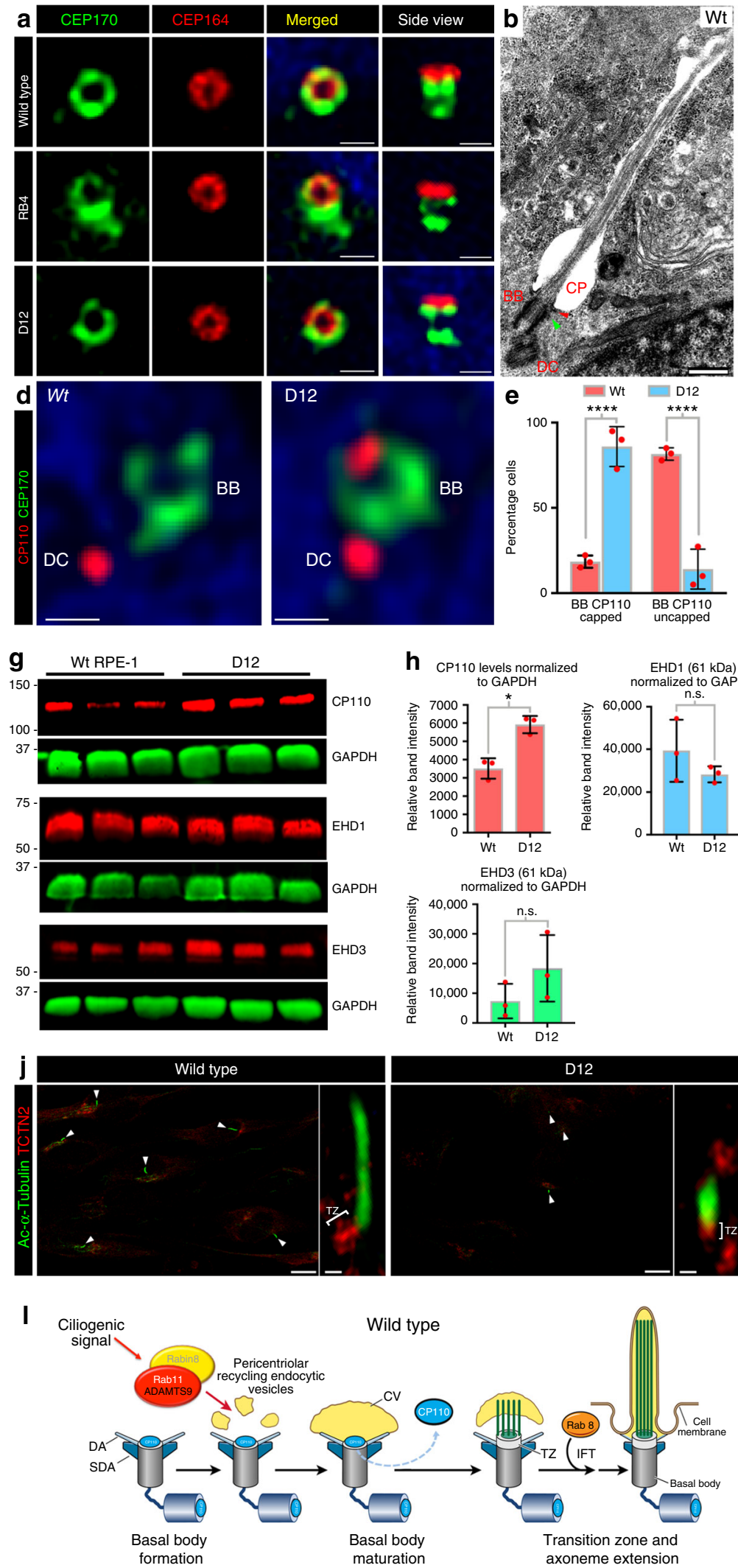

h
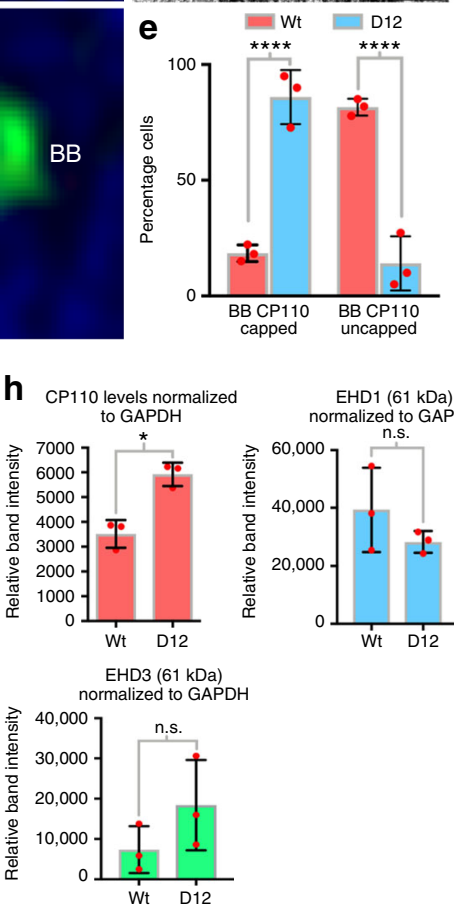
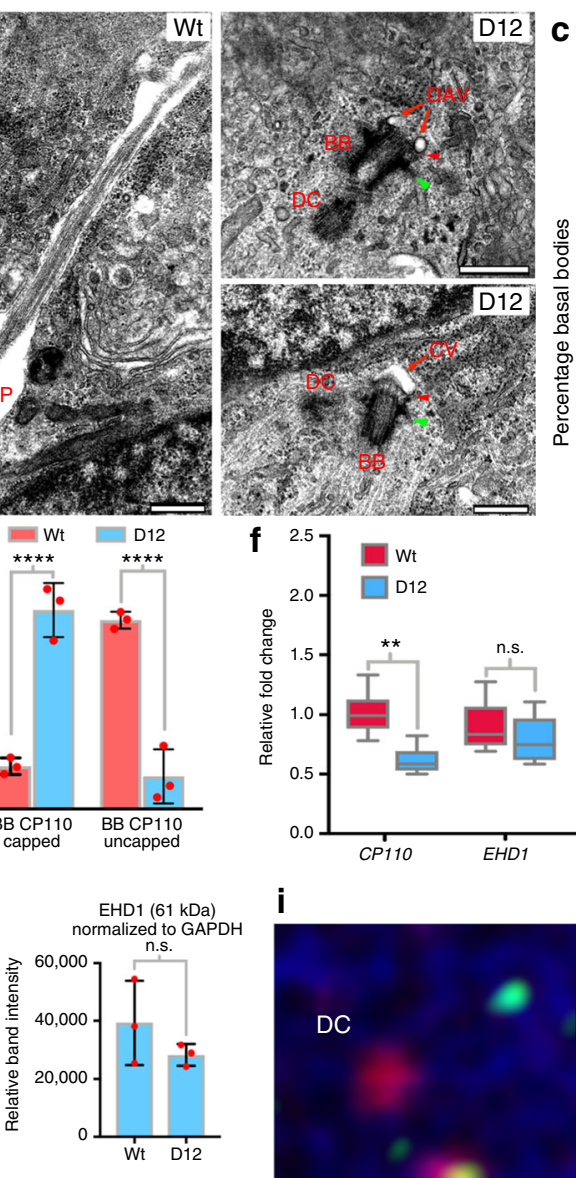

C $\square$ Extended axoneme
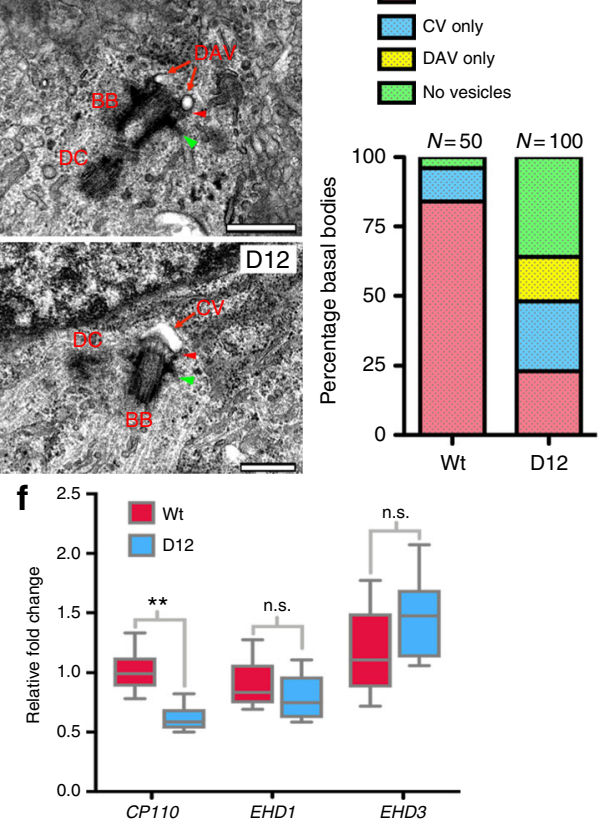

i
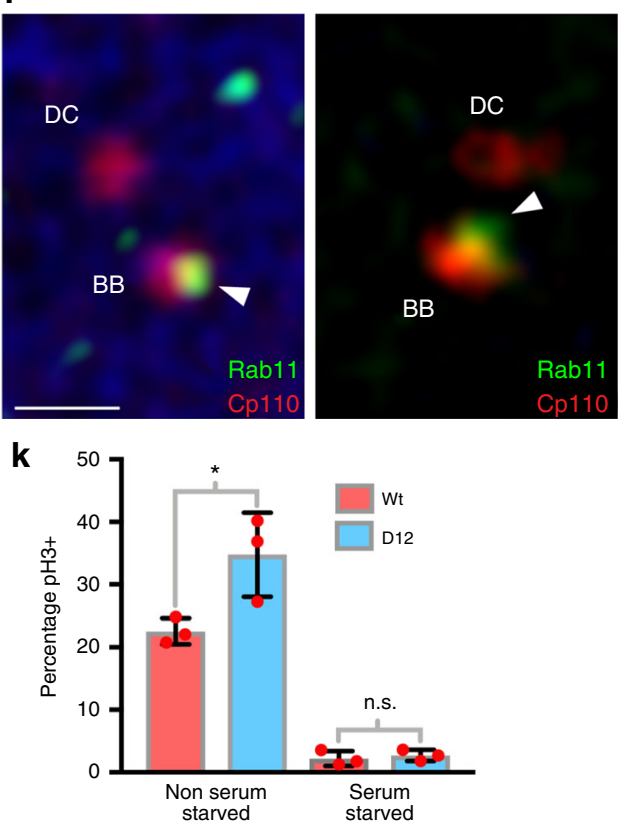

m

$$
\text { Adamts }{ }^{/-}
$$

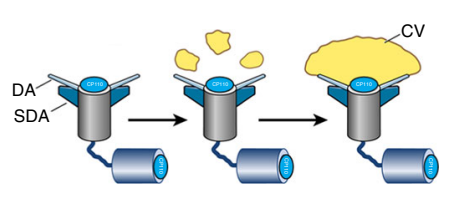

sac, where Adamts20 is not expressed, deficiency of Adamts 9 alone impairs ciliogenesis and when Adamts9 dosage was substantially reduced below that of Adamts $9 \mathrm{Gt} / \mathrm{Gt}$ embryos in Adamts 9 Gt/Del embryos, open neural tube and defective cilia also resulted. We conclude that many cells may be over-engineered to ensure availability of ADAMTS9 and/or ADAMTS20 above a required threshold, and that both proteases may be broadly relevant to ciliogenesis. Indeed, ADAMTS9 was previously identified as a positive regulator of ciliogenesis in an siRNA screen for putative ciliary length regulators 7 , but not investigated further and has been recently implicated as the mutant gene in human ciliopathies affecting the kidney and brain ${ }^{54}$. 
Fig. 4 Basal body maturation is impaired in ADAMTS9-null cells. a Super-resolution imaging of distal (CEP164, red) and sub-distal appendages (CEP170, green) shows normal basal body formation in RB4 and D12 cells. b TEM of serum-starved wild-type and D12 cells shows D12 basal bodies (BB) with docked distal appendage vesicles (DAV) and ciliary vesicles (CV) (red arrows) but no axoneme. CP, ciliary pocket; DC, daughter centriole. c Quantification of basal body-associated vesicles at different stages of formation and growth shows that the majority of the mutant basal bodies do not progress beyond ciliary vesicle docking. $N=50 \mathrm{BB}(\mathrm{WT}), \mathrm{N}=100 \mathrm{BB}$ (D12). d DSCM imaging of $24 \mathrm{~h}$ serum-starved wild-type and D12 cells stained for CP110 (red) and CEP170 (green) shows that the basal body of D12 cells retains the distal CP110 cap. e Quantification of basal bodies with and without CP110 caps ( $N=3$ independent experiments, ${ }^{\star \star \star \star} p<0.00001$, Student's $t$ test). f Quantitative RT-PCR of CP110, EHD1 and EHD3 mRNAs in wild-type (Wt) and D12 RPE1 cells. $N=3$ independent experiments; n.s., not significant; ${ }^{\star \star} p<0.001$, Student's $t$ test. $\mathbf{g}$, h Western blot (g) and quantification (h) of CP110, EHD1 and EHD3 (red) normalized to GAPDH (green) shows higher CP110 in $24 \mathrm{~h}$ serum-starved ADAMTS9 mutant D12 cells but no change in EHD1/ EHD3 levels ( $N=3$ independent experiments, ${ }^{\star} p<0.05$, Student's $t$ test). i DSCM imaging of $6 \mathrm{~h}$ serum-starved wild-type RPE-1 cells co-stained with CP110 (red) and Rab11 (green) shows Rab11 + vesicles near CP110 cap of the basal bodies (arrowheads). j Staining for the transition zone (TZ) marker TCTN2 shows a TZ in wild type cells and the few mutant (D12) cells that produce ciliary axonemes (arrowheads). k Quantification of phospho-histone H3 positive cells show increased cell proliferation in non-serum-starved mutant cells and minimal proliferation upon serum starvation, similar to wild-type cells $(N=3$ experiments, ${ }^{*} p<0.05$, Student's $t$ test). I, $\mathbf{m}$ Schematic showing the ciliogenesis sequence ${ }^{33}$ and arrest at the ciliary vesicle docking stage in the absence of ADAMTS9 (right). Scale bars $=500 \mathrm{~nm}$ in a-d, i. Bar charts and dotplots show mean and SD (error bars). In box and whisker plots, whiskers indicate the range and boxes mark upper and lower quartiles. The centerline indicates the mean
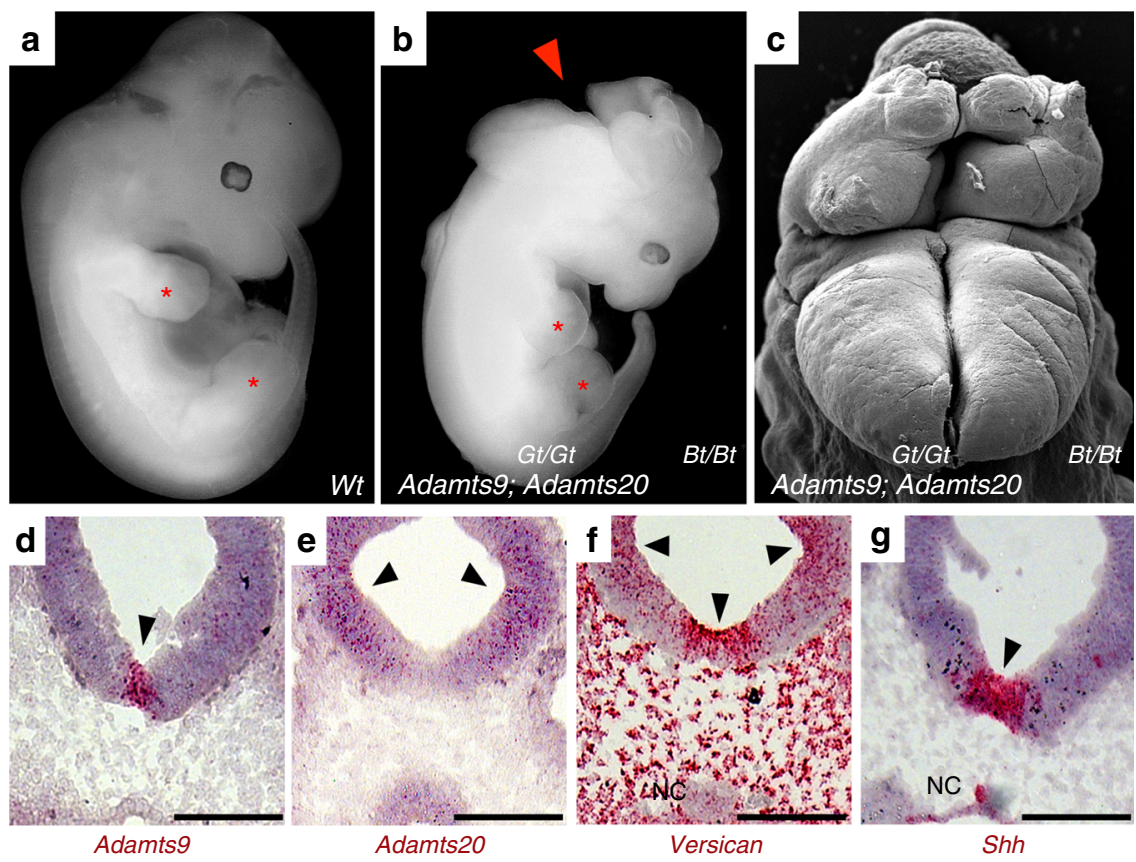

Fig. 5 Severe developmental defects resembling ciliopathies in Adamts9Gt/Gt; Adamts20 ${ }^{B t / B t}$ mouse embryos. a E12.5 wild-type and Adamts9Gt/Gt; Adamts $20^{B t} / B t$ embryos (b) showing occipital exencephaly (red arrowhead) and a malrotated body axis in the latter. Red asterisks indicate right-side limbs, which are abnormally close in the mutant (b). c Scanning electron micrograph of the exencephalic hindbrain of an E12.5 Adamts9Gt/Gt;Adamts20Bt/Bt embryo. d-g RNAscope in situ hybridization shows expression domains of Adamts9 (d), Adamts20 (e), their cognate ECM substrate Vcan (f) and Shh (g) in the rostral neural epithelium of E9.5 embryos. Robust Adamts9 expression is observed in the rostral floor plate (arrowhead in d) within the Shh expression domain (arrowhead in $\mathbf{g}$ ). Adamts20 is predominantly expressed in the lateral neural epithelium (arrowheads in $\mathbf{e}$ ). Vcan is strongly expressed in the floor plate and the lateral neural epithelium (lateral arrowheads in $\mathbf{f}$ ), with strong expression in surrounding craniofacial mesenchyme. NC, notochord. Scale bars $=100 \mu \mathrm{m}$ in $\mathbf{d}-\mathbf{g}$

Incorporating prior work on ADAMTS9 biosynthesis and trafficking $12,17,20,55-58$ as well as new data on heparin binding and receptor-mediated endocytosis, the present work tracks the voyage of ADAMTS9 from the secretory pathway to cilium (Fig. 10a). ADAMTS9, having undergone obligate, regulatory glycosylation in the secretory pathway ${ }^{56,58}$, binds to the cell surface, which may be via HSPG, LRP1/2 and furin interaction $^{12,20}$ and is activated by furin ${ }^{20}$. It is then recycled to the ciliary vesicles via LRP1/2 and clathrin-mediated endocytosis to regulate ciliogenesis via accumulation around the basal body (Fig. 10b). ADAMTS20 has not been as extensively investigated, but the limited data clearly suggest that it too is internalized to participate in ciliogenesis.
Considerable evidence argues against unconventional ADAMTS9 transfer into the cytosol. ADAMTS9 has a bona fide signal peptide and transfected ADAMTS9 constructs are readily detected at the cell surface and in the medium ${ }^{12,56,58}$, bound to secretory chaperones ${ }^{57}$ and modified by Golgi enzymes ${ }^{20,56,58}$. That ADAMTS9 present at the cilium base lacks its propeptide strongly supports recycling of secreted ADAMTS9, since its propeptide is excised at the cell surface and not in the transGolgi $^{20}$. Notably, CHC-knockdown or inhibition of clathrinmediated endocytosis removed ADAMTS9 from the cilium base. Also arguing against an alternative mRNA spliced cytosolic ADAMTS9 proteoform, ciliogenesis was rescued in D12 cells by transfection with the canonical ADAMTS9 and ADAMTS20 

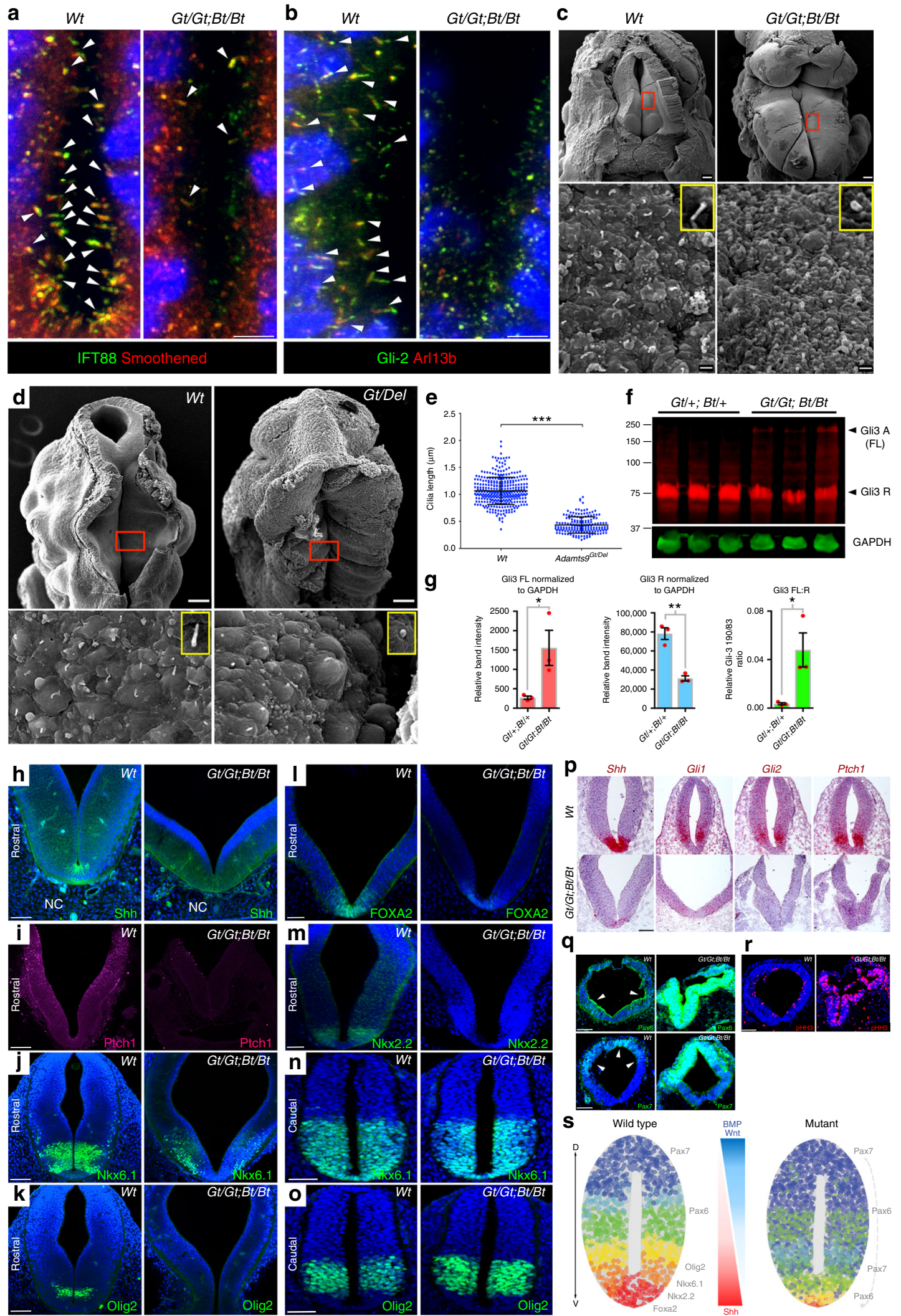

cDNA. ADAMTS9Gt arose from a gene trap insertion that leads to constitutive ADAMTS9 membrane anchorage with impaired furin processing ${ }^{17}$. Since ADAMTS9Gt/Gt yolk sac mesothelium lacks cilia, we conclude that release from the cell and propeptide excision by furin are crucial for ADAMTS9 function. The rescue of ciliogenesis in ADAMTS9-deficient RPE1 cells by
ADAMTS9 supplied in conditioned medium and localization of exogenous ADAMTS9 to the cilium base conclusively establishes the constitutively secreted protein as the participant in ciliogenesis.

Adamts9 and Adamts20 mRNA expression domains had minimal overlap in the neural tube, yet Adamts $9^{\mathrm{Gt} / \mathrm{Gt}}$ embryos 
Fig. 6 Adamts9Gt/Gt; Adamts20Bt/Bt embryos have impaired ciliogenesis and Shh signaling. a, b Coimmunostaining for IFT88 (green) and Arl13b (red) with smoothened (red), and Gli-2 (green) respectively, reveals fewer cilia and reduced Hh signaling in E9.5 Adamts9Gt/Gt; Adamts20Bt/Bt neural tubes. c Scanning electron microscopy (SEM) of E12.5 wild type and Adamts9Gt/Gt; Adamts20Bt/Bt neural tubes shows short cilia in mutants. Boxed areas are shown at higher magnification in lower panels. Inserts (yellow) in the lower panels show single cilia. d SEM of E9.5 Adamts9Gt/Del embryos shows an open neural tube and short cilia. Red boxes are shown at higher magnification in the lower panels. Inserts in the lower panels (yellow box) show single cilia. e Quantification of primary cilium length in wild-type and Adamts $9 \mathrm{Gt} / \mathrm{Del}$ embryos shows short cilia in the mutants $\left(N=3 \mathrm{embryos}\right.$, ${ }^{\star \star \star} p<0.001$, Student's $t$ test). f Gli3 western blot of E12.5 Adamts9Gt/+; Adamts20Bt/+ and Adamts9Gt/Gt; Adamts20 ${ }^{B t / B t}$ heads shows reduced processed Gli3 ( $83 \mathrm{kDa}$ ) in mutants. g Quantification of relative band intensities for full-length $\mathrm{Gli3}(190 \mathrm{kDa})$ and processed $\mathrm{Gli} 3(83 \mathrm{kDa})$ form and the relative ratios $\left(\mathrm{N}=3 \mathrm{embryos}\right.$, ${ }^{*} p<$ $0.05,{ }^{* *} p<0.001$, Student's $t$ test). h-m Rostral neural tube sections of E9.5 wild-type and Adamts9Gt/Gt; Adamts20Bt/Bt embryos immunostained for Shh $(\mathbf{h})$, Ptch1 (i), Nkx6.1 (j), and Olig-2 (k), FOXA2 (I), Nkx2.2 ( $\mathbf{m})$ shows reduced Shh signaling and aberrant ventralization in mutants $(N=3$ embryos each). $\mathrm{NC}$, notochord. $\mathbf{n}$, o Nkx6.1 (n) and Olig-2 (o) staining (green) of caudal neural tube sections show normal Shh signaling caudally in mutants ( $\mathrm{N}=3$ embryos each). p ISH for Shh, Gli1, Gli2 and Ptch1 mRNA shows reduced Shh-responsive mRNAs in Adamts9Gt/Gt; Adamts20 ${ }^{B t / B t}$ rostral neural tube ( $N=3$ embryos each for each probe). q BMP-activated dorsal neuronal markers Pax6 and Pax7 (green) show stronger signal and ventral expansion in Adamts9Gt/

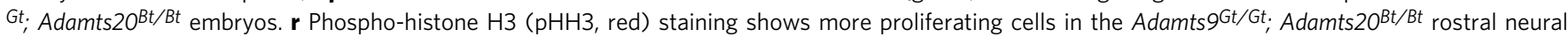
tube. s Cartoon depicting neuronal domains in wild-type and mutant neural tubes. Scale bars $=50 \mu \mathrm{m}$ in $\mathbf{h}-\mathbf{r}, 5 \mu \mathrm{m}$ in $\mathbf{a}, \mathbf{b}$ and $100 \mu \mathrm{m}$ and $1 \mu \mathrm{m}$ in $\mathbf{c}$, d. Bar charts and dotplots show mean (centerline) and SD (error bars) except $\mathbf{g}$ (S.E.M.)

completed neural tube closure. Here, it is possible that low-level Adamts20 expression, or secreted ADAMTS20 acting in-trans compensates for reduced ADAMTS9 activity. Indeed, in RPE1 cells, the present work unequivocally demonstrates that ADAMTS9 and ADAMTS20 are interchangeable, and can act in trans. ADAMTS9 and ADAMTS20 catalytic activity is essential for their function at the ciliary base, but whether it functions incis, i.e., via autocatalysis of each protease to a functionally optimal product essential for ciliogenesis, or by proteolysis of an intravesicular substrate to activate its role in ciliogenesis remains to be elucidated. In theory, ADAMTS9/ADAMTS20 localized to the Rab11+ vesicles, could proteolytically modify the ectodomain of a transmembrane molecule necessary for ciliogenesis.

The role of endocytic trafficking in ciliary vesicle development is poorly understood and few secreted proteins participating in ciliogenesis are known. A seminal study of ciliogenesis identified a ciliary vesicle adjacent to the nascent axoneme and determined that it was of Golgi origin ${ }^{59}$, yet recycling endosome markers also label peri-centrosomal vesicles ${ }^{7}$. Knockdown of PTPN23, a nontransmembrane tyrosine phosphatase required for endosomal cargo sorting in early endocytic compartments ${ }^{60}$, and ASAP1, a GTPase-activating protein required for peri-centrosomal accumulation of endocytic vesicles ${ }^{61}$, significantly reduced the number of ciliated RPE-1 cells. A secreted phospholipase PLA2G3, is a negative ciliogenesis regulator ${ }^{7}$ and PI3K-C2- $\alpha$, a phosphatidylinositol 3-kinase, regulates endocytic trafficking to the cilium and ciliogenesis $^{62}$. To our knowledge the only other secreted molecule associated with a cilium defect is laminin-511 63 . Although PTPN23, PI3K-C2- $\alpha$ and PLA2G3 localize in peri-centromeric endocytic vesicles ${ }^{7,62}$, laminin-511 is not known to do so and may act indirectly by ensuring apical-basal polarity of epithelial cells, which manifest short primary cilia in its absence ${ }^{63}$. ADAMTS9 is concentrated in a distinct subset of Rab11+ late-recycling endocytic vesicles surrounding the basal body. Together, with Rab8, a master regulator of ciliary protein trafficking, Rab11+ vesicles transport cargo bound for the cilium during ciliogenesis (reviewed by ref. ${ }^{64}$ ). ADAMTS9-containing vesicles comprise a unique population lying external to previously identified concentric domains surrounding the centrosome. ADAMTS20 trafficking was not investigated in the same detail, since its ability to rescue of ciliogenesis in the D12 cells and comparable localization as ADAMTS9 to the cilium base suggests it follows a similar pathway.

ADAMTS9 and ADAMTS20 cooperate with ADAMTS5 in versican proteolysis during interdigit web regression ${ }^{18}$, but ADAMTS5 did not rescue the cilium defect in D12 cells, suggesting that it was not directed to the Rab11+ vesicle population or it lacks the specific proteolytic activity embodied in ADAMTS9 and ADAMTS20. ADAMTS16, which is distantly related to ADAMTS9, was reported to restrict the length of the primary cilium in renal tubular epithelium ${ }^{53}$; however, its loss had no impact on rat morphogenesis, and it is not known to localize to the cilium. Although the present studies unequivocally demonstrate the role of ADAMTS9 and ADAMTS20 in ciliogenesis, they could contribute to neural tube closure and Hh signaling in mice by additional mechanisms. ADAMTS9 heparin-binding is potentially relevant to Shh activation, diffusion or transport, since Shh binds HSPGs and undergoes proteolytic shedding at the cell surface $^{65,66}$. Furthermore, increased proteoglycan in neural epithelium can potentially modify tissue biophysical properties and cell adhesion, each being required for closure of the neural tube ${ }^{67}$. We have not formally tested these possibilities, choosing to specifically address ciliogenesis here.

A key question that arises is whether the observed primary cilium defect is secondary to impaired ECM turnover. The cilium is closely approximated to ECM and carries ECM receptors such as integrins ${ }^{68}$. Altered cell-matrix interactions in the absence of ADAMTS9 influence smooth muscle cell focal adhesion formation, shape, actin cytoskeleton assembly and nuclear morphology via accumulation of pericellular versican ${ }^{69}$. Arguing for a direct role in ciliogenesis, ADAMTS9 and ADAMTS20 localization to peri-centrosomal vesicles is necessary for ciliogenesis. RPE-1 cells express low levels of the ADAMTS substrate versican, and no changes in versican or cell shape (reflecting anomalous cell - matrix interactions) were noted in D12 cells in the $24 \mathrm{~h}$ timeframe utilized in our ciliogenesis assays. Furthermore, expression of ADAMTS5 did not rescue ciliogenesis in ADAMTS9-deficient D12 cells, versican overexpression did not affect ciliogenesis in wild-type RPE-1 cells, and the ciliogenesis defect of D12 cells could not be rectified by providing a high concentration of the proadhesive molecule fibronectin. We therefore conclude that although ADAMTS9 and ADAMTS20 unequivocally mediate proteolytic modification of ECM components as a canonical function, they act noncanonically as modulators of ciliogenesis. Major future directions from this work include identification of potential ADAMTS9/ADAMTS20 substrates at either the cell surface or within cilia-bound endocytic vesicles that are crucial for ciliogenesis.

\section{Methods}

Mice. Mouse experiments were conducted under a Cleveland Clinic Institutional Animal Care and Use Committee (IACUC) approved protocol (2015:1530), in compliance with all relevant ethical regulations. Mice were maintained in a fixed light-dark cycle with ad libitum access to food and water. Adamts ${ }^{G t}{ }^{17}$,

Adamts $9^{\mathrm{Del} 45}$, Adamts $20^{\text {Bt } 13}$ and Vcan ${ }^{\text {Hdf } 70}$ alleles and their genotyping were 

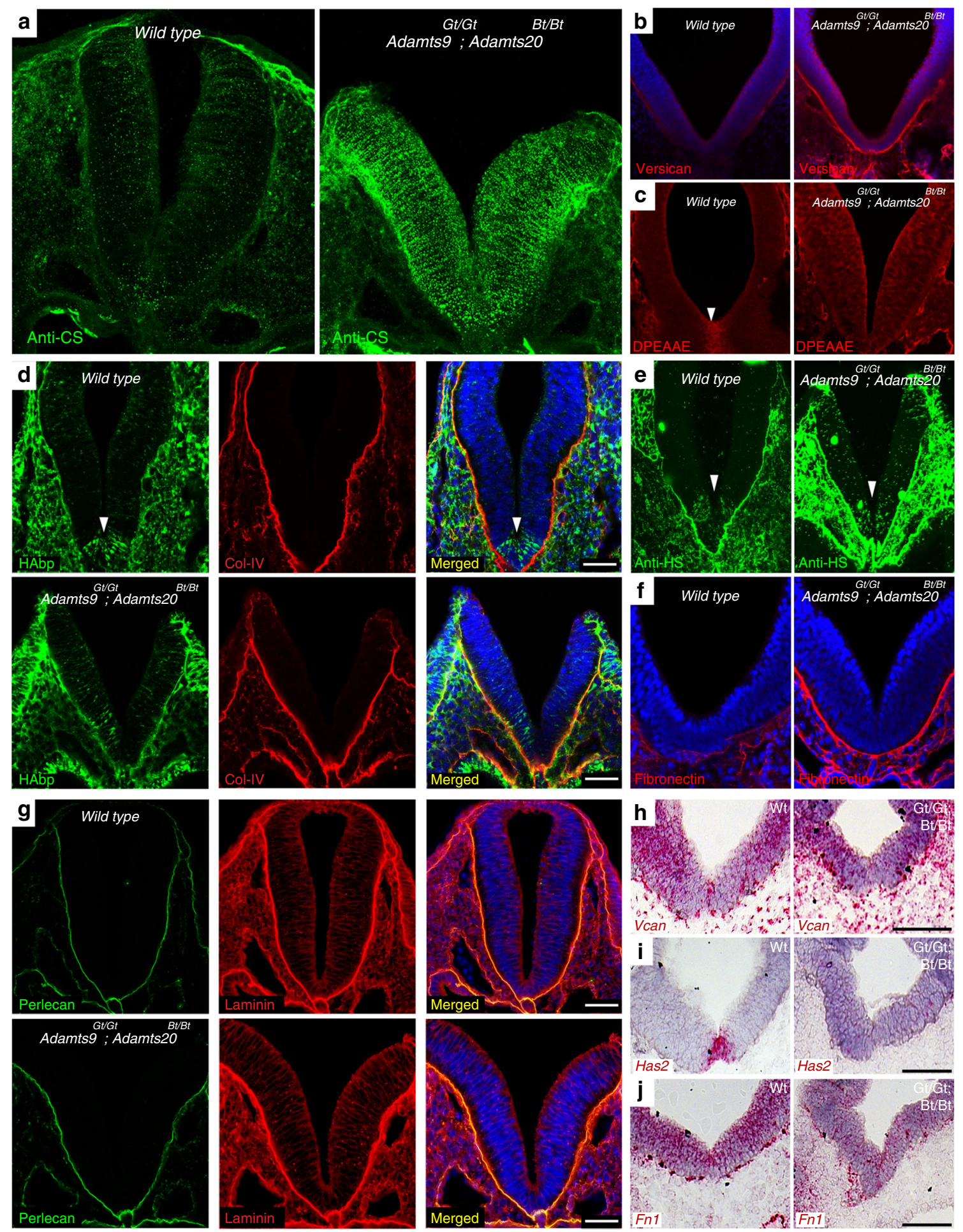

Fig. 7 Abnormal ECM dynamics in Adamts9Gt/Gt; Adamts20Bt/Bt neural epithelium. a 3D-projection of $30 \mu m$-thick vibratome sections stained for chondroitin sulfate (CS) in E9.5 wild-type and Adamts9Gt/Gt; Adamts20 2 Bt/Bt embryos shows intense CS staining in interstitial ECM of mutant neural epithelium. b Immunostaining for versican (GAG $\beta$ ) shows stronger staining throughout mutant neural epithelium. c The versican-neo epitope antibody anti-DPEAAE shows strong staining in the midline of the wild-type neural tube floor plate (arrowhead) but not in the mutant. $\mathbf{d}$ Coimmunostaining with hyaluronan-binding protein (HAbp) (green) and anti-collagen IV (red) shows loss of hyaluronan in the mutant floor plate. Collagen IV in the basement membrane of the neural epithelium shows no change in the mutant. e Anti-heparan sulfate (anti-HS) shows intense staining in the mutant craniofacial mesenchyme around the neural epithelium and to a lesser degree in the neural tube (arrowhead). $\mathbf{f}$ Fibronectin is present in wild-type neural tube basement membrane and surrounding mesenchyme where it stains strongly in the mutant. $\mathbf{g}$ Perlecan (green) and laminin (red) show comparable staining of wild-type and mutant basement membranes. Laminin is also present in ECM of the craniofacial mesenchyme. $\mathbf{h}$-j RNA in situ hybridization shows loss of Has2 $(\mathbf{i})$ in the mutant neural tube whereas $V$ can $(\mathbf{h})$ and $F n 1(\mathbf{j})$ expression are unaffected. Scale bars $=50 \mu \mathrm{m}$ in $(\mathbf{d}-\mathbf{g}), 100 \mu \mathrm{m}$ in $(\mathbf{h}-\mathbf{j})$ 

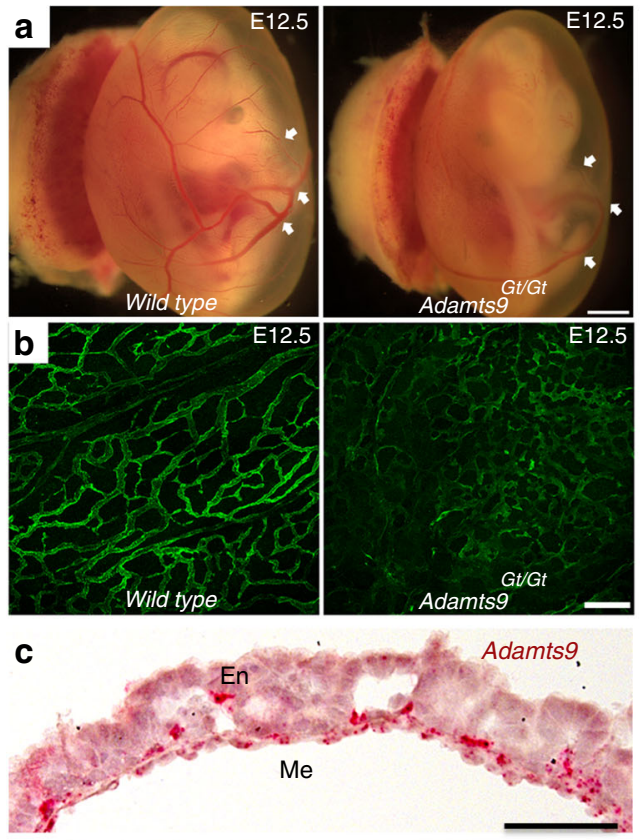

d
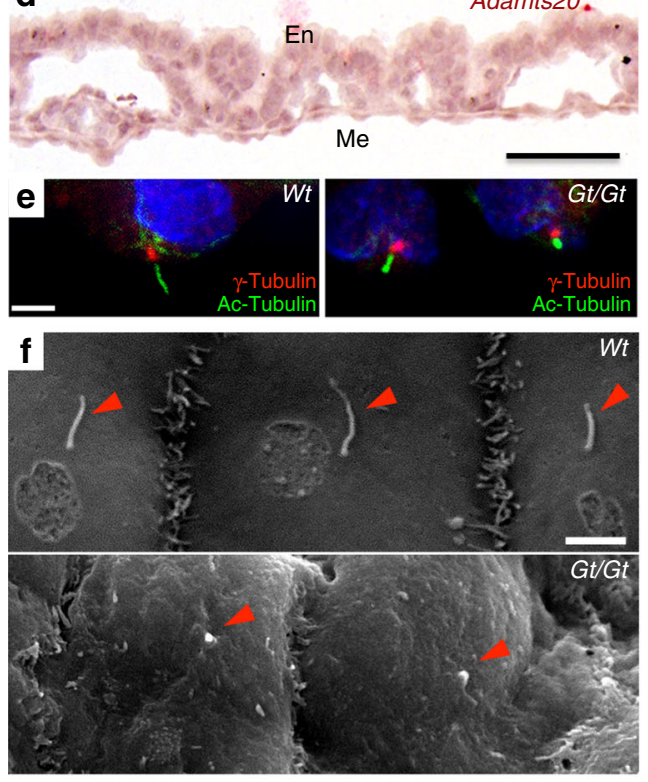

g

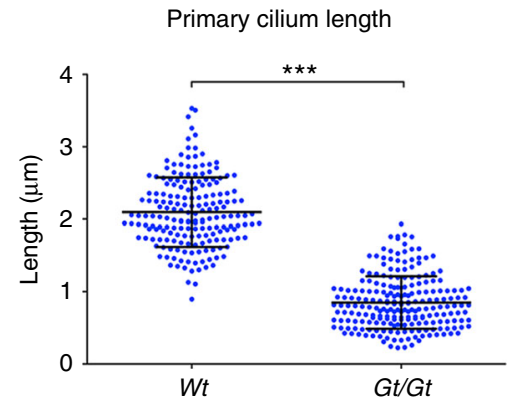

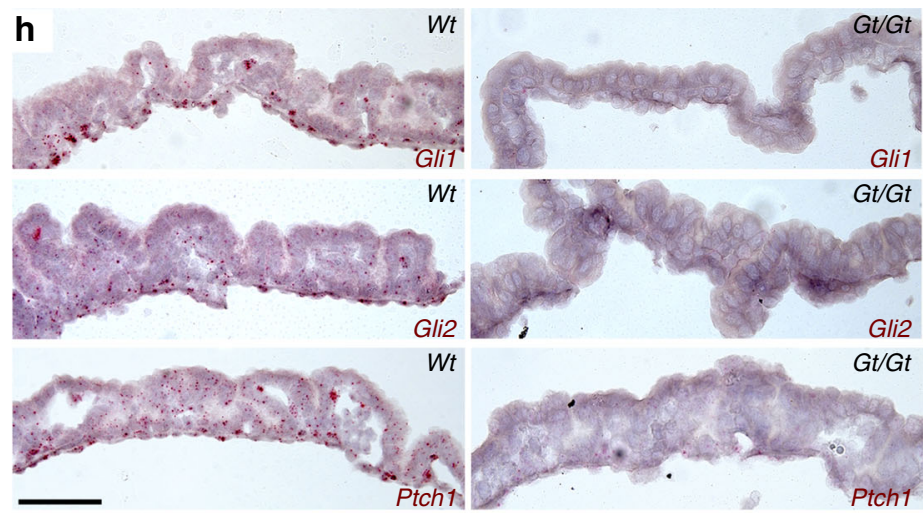
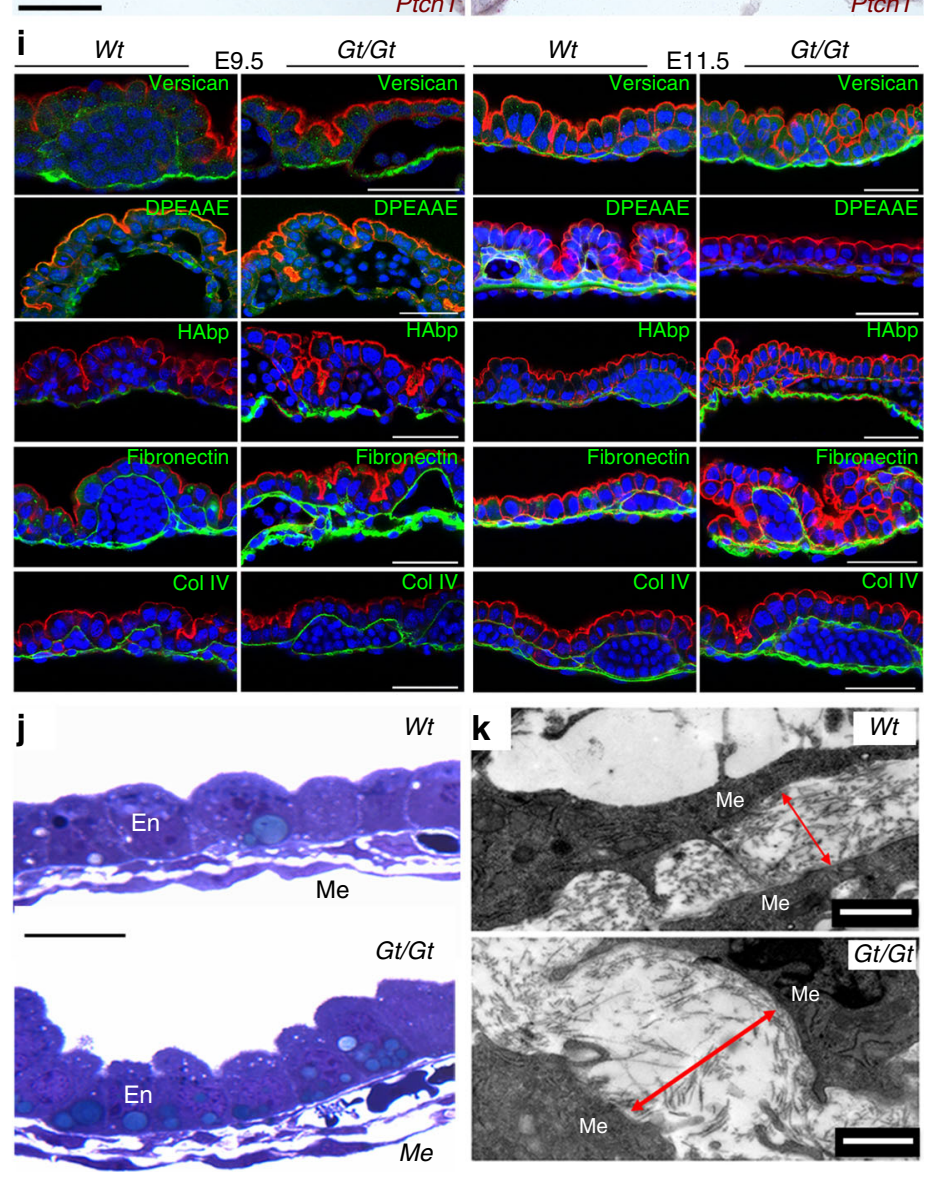

I

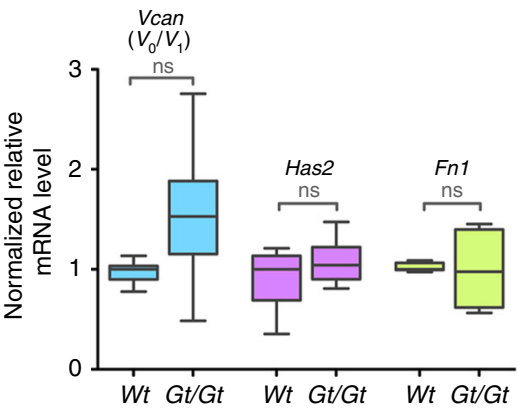

previously described. Ptch $1^{\text {LacZ }}$ mice (Stock No. 003081) and ShheGFP-Cre mice (Stock No. 005622) were purchased from The Jackson Laboratories and genotyped as instructed.

Mammalian cell culture. hTERT RPE-1 (CRL-4000; ATCC, Manassas, VA), IMCD-3 (CRL-2123; ATCC, Manassus, VA), and NIH-3T3 cells (CRL-1658; ATCC, Manassus, VA) were cultured in DMEM F12 with $10 \%$ FBS, $100 \mathrm{U} / \mathrm{mL}$ penicillin/streptomycin under $5 \% \mathrm{CO}_{2}$. RPE-1 growth medium was supplemented with $0.01 \mathrm{mg} / \mathrm{mL}$ hygromycin $\mathrm{B}$. Human neonatal foreskin dermal fibroblasts (HDFs) were purchased from Lonza (CC-2509, Basel, Switzerland) and cultured in DMEM with $10 \% \mathrm{FBS}, 100 \mathrm{U} / \mathrm{mL}$ penicillin/streptomycin under $5 \% \mathrm{CO}_{2}$.

Plasmids and transfection. Expression plasmids for human ADAMTS9, mouse ADAMTS20, and their corresponding inactive mutants (ADAMTS9 E/A and 
Fig. 8 Defective angiogenesis, cilia, Hh signaling and ECM in Adamts9Gt/Gt yolk sac. a E12.5 Adamts9Gt/Gt embryos have poorly formed yolk sac vasculature. White arrows point to vitelline vessel branch points. $\mathbf{b}$ En-face images of isolectin-B4-stained (green) whole-mount yolk sacs show abnormal vasculature in Adamts9Gt/Gt yolk sacs. c, d RNA in-situ hybridization shows robust Adamts9 expression (red) in E11.5 mesoderm (ME) but not yolk sac endoderm (En). Adamts20 (d) is not expressed in the yolk sac. e Primary cilia and centrosomes visualized by acetylated $\alpha$-tubulin (green) and $\gamma$-tubulin (red) respectively, or SEM (f, arrowheads) show short bulbous-tipped primary cilia in Adamts $9 \mathrm{Gt} / \mathrm{Gt}$ yolk sac mesothelium. $\mathbf{g}$ Quantitation of primary cilium length by SEM in wildtype and Adamts9Gt/Gt yolk sac mesothelium ( $N=3$ yolk sacs from each genotype, ${ }^{\star \star \star} p<0.001$, Student's $t$ test). $\mathbf{h}$ In-situ hybridization for Gli1, Gli2, and Ptch1 mRNA in E11.5 yolk sacs (red) shows absent hedgehog signaling in Adamts9Gt/Gt yolk sacs ( $N=3$ yolk sacs from each genotype). i E9.5 and E11.5 Adamts 9 Gt/Gt yolk sacs were stained for versican, cleaved versican (DPEAAE), hyaluronan (HAbp), fibronectin, and collagen-IV, and counterstained with Alexa-568 phalloidin (red) to outline cell layers. Versican, hyaluronan and fibronectin accumulated in yolk sac mesoderm whereas staining for versican neoepitope DPEAAE was lost in the Adamts $9 \mathrm{Gt} / \mathrm{Gt}$ yolk sac. Collagen IV staining was unaffected ( $N=3$ yolk sacs from each genotype). $\mathbf{j}$ Toluidine blue staining of $1 \mu \mathrm{m}$ plastic-embedded sections of E11.5 yolk sacs show a thicker mesoderm in the mutant resulting from expanded ECM. $\mathbf{k}$ TEM images of the yolk sac show increased ECM between mesoderm cells (red arrows) in the Adamts $9 \mathrm{Gt} / \mathrm{Gt}$ yolk sac, primarily noncollagenous in appearance. I qRT-PCR analysis for $V$ can, Has2 and Fn1 shows comparable levels in mutant and wild-type yolk sac ( $N=3$ yolk sacs from each genotype, Student's $t$ test). Scale bars $=2 \mathrm{~mm}$ in $\mathbf{a}, 100 \mu \mathrm{m}$ in $\mathbf{b}, 50 \mu \mathrm{m}$ in $\mathbf{c}, \mathbf{d}$ and $\mathbf{h}, \mathbf{i}, 2 \mu \mathrm{m}$ in $\mathbf{e}, \mathbf{f}, 10 \mu \mathrm{m}$ in $\mathbf{j}, \mathbf{k}$, and $1 \mu \mathrm{m}$ in the lower panels of $\mathbf{k}$. Dotplot shows mean and SD. In box and whisker plot, whiskers indicate the range and boxes mark upper and lower quartiles. The centerline indicates the mean

ADAMTS20 E/A) were previously described ${ }^{12,20}$ as were ADAMTS1, ADAMTS5, ADAMTS16 and TIMP3 constructs $^{71-74}$. Three hundred nanograms of each construct was transfected using Lipofectamine 3000 reagent (Invitrogen, catalog no. L3000-015) in eight-well chamber slides. Fifty nanograms of Vcan-V1 plasmid ${ }^{75}$ was transfected for over expressing versican in RPE-1 cells.

CRISPR/Cas9 mutagenesis. We used custom polycistronic plasmids encoding guide RNAs targeting exon 2 of ADAMTS9, Cas9 and GFP or RFP (Sigma-Aldrich, Cat. No. 03211705MN, 03211707MN). For establishing mutant cell lines, hTERTRPE-1 cells were cultured in six-well plates and transfected with $2.5 \mu \mathrm{g}$ of plasmid DNA using Lipofectamine 3000 reagent (Invitrogen, Cat. No. L3000-015). Transfected cells were trypsinized after $24 \mathrm{~h}$ and GFP or RFP-positive cells were sorted into 96-well plates using- the FACSAria-II cell sorter for clonal growth (BD Biosciences). Wells containing $>1000$ cells/clone were trypsinized and expanded into 24-well plates after 1 week. Genomic DNA was harvested from the established clones using the DirectPCR (Tail) digest reagent (Viagen, Cat. No. 102-T) and exon 2 of ADAMTS9 was PCR amplified using the proof-reading Phusion high-fidelity polymerase (NEB, Cat. No. F530L). Amplicons were excised from $2 \%$ agarose gels, purified using the QIAquick Gel Extraction kit (QIAGEN, Cat. No. 28704), cloned in pCR-Blunt II-TOPO vector using the Zero blunt PCR cloning kit (Life Technologies, Invitrogen, Cat. No. K2800-40) and transformed into One Shot TOP10 chemically competent $E$. coli (Invitrogen, Cat. No. C404006). At least ten bacterial colonies grown on LB agar plates containing $50 \mu \mathrm{g} / \mathrm{mL}$ kanamycin were picked (corresponding to a single 96-well RPE-1 cell colony). Plasmid DNA was harvested using the QIAprep spin mini-prep kit and the inserted amplicons were Sangersequenced using the SP6 primer. hTERT-RPE1 clones with 50\% disrupted and 50\% wild-type sequences were considered heterozygous (clone RB4), whereas those with $100 \%$ mutant sequences were identified as homozygous mutants (D12). Lack of ADAMTS9 was confirmed by western blotting and immunostaining.

Immunostaining and fluorescence microscopy. Immunostaining of mouse tissue was carried out on $30 \mu \mathrm{m}$-thick vibratome sections ${ }^{17}$, or on paraffin-embedded 7 $\mu \mathrm{m}$ sections. Immunostaining of cultured cell lines were carried out in fourchamber or eight-chamber cell culture slides. High-resolution fluorescent images of mouse embryo sections were acquired using a Leica TCS SP5 II multiphoton confocal microscope equipped with a $\times 25$ water immersion objective (Leica Microsystems, Wetzlar, Germany) or using an Olympus BX51 upright microscope (Olympus, Center Valley, PA) equipped with a Leica DFC7000T camera and Leica Application Suite v4.6 imaging software (Leica Microsystems, Wetzlar, Germany). Details of all antibodies used are provided in Supplementary Table 1.

\section{Deconvolution super-resolution confocal microscopy (DSCM). A Leica TCS} SP8 confocal microscope (Leica Microsystems, Wetzlar, Germany) upgraded with the Hyvolution-2 modification was used for super-resolution microscopy. In brief, confocal images were acquired utilizing the HyD hybrid detectors, and the Leica LAS X operating software (version 3.1.5.16308) and simultaneously deconvolved using the Huygens HyVolution-2 plug-in (Scientific volume Imaging, Hilversum, Netherlands). Hyvolution-2 combines multiparameter fluorescence imaging with point spread function-based real deconvolution, allowing for high-speed multicolor super-resolution imaging with a maximum resolution of $140 \mathrm{~nm}$. $\times 1000$ superresolution images of primary cilia or centrosomes in RPE-1 cells were acquired using an HCX PL Apo $\times 100$ 1.47NA oil immersion objective and a $\times 10$ zoom. Cells were cultured in Falcon 4-chamber slides (Fisher scientific, Cat. No. 354114,) and mounted in ProLong Gold Antifade mountant containing DAPI (Cell Signaling, Cat. No. 8961S). For 3D-projection of deconvoluted Z-stacks, the Volocity 3D imaging software (version 6.3, PerkinElmer, Inc., Waltham, MA) 3D-opacity projection method was used.

Optical projection tomography. Fixed embryos were embedded in low melting point agarose (IBI scientific, Cat. No IB70056) (1.1\% in Milli-Q water), the agarose blocks trimmed and then superglued to magnetic stubs. The embedded tissue was then placed in $100 \%$ methanol for $24 \mathrm{~h}$, which was replaced twice (each for a further $24 \mathrm{~h}$ ), to ensure complete dehydration. The samples were submerged in one part benzyl alcohol to two parts benzyl benzoate for $24 \mathrm{~h}$ and again the solution replaced twice for $24 \mathrm{~h}$ each to ensure complete optical clearing of the tissue. Cleared samples were subjected to imaging in a Bioptonics 3001M optical Projection Tomograph in the Small Animal Tomographic Analysis (SANTA) Facility at the Seattle Children's Research Institute. The embryos were imaged at $\sim 12.6 \mu \mathrm{m}$ resolution using a $1024 \times 1024$ image format: $88 \mathrm{~ms}$ exposure, three-frame averaging. Imaging was conducted under UV light using a GFP1 filter (425 nm excitation, $475 \mathrm{~nm}$ emission) to detect tissue autofluorescence. Raw imaging data (TIF format) were reconstructed into multidimensional slice data (BMP format) using NRecon v1.6 software (Skyscan, Belgium). Reconstructed data were then analyzed both in 2D multiplanar format in Dataviewer (Skyscan, Belgium) and in 3D following rendering in the Drishti v2.6 Volume Exploration software (http://sf.anu. edu.au/Vizlab/drishti).

Electron microscopy and immunogold electron microscopy. For SEM imaging of neural tube or yolk sac cilia, embryos were harvested from timed pregnancies a 9.5 or at 12.5 days of gestation and fixed in $4 \%$ paraformaldehyde (PFA) $+2.5 \%$ glutaraldehyde in PBS overnight. To visualize primary cilia in wild-type neural tube, the cranial vault was de-roofed after fixation. Samples were post-fixed in $1 \%$ osmium tetroxide for $1 \mathrm{~h}$, washed in deionized sterile water and dehydrated in an ethanol gradient. Samples were immersed in a 1:1 mixture of 100\% EtOH:100\% hexamethyldisilazane (HMDS) overnight, washed three times in 100\% HMDS and air dried for $48 \mathrm{~h}$. Dehydrated samples were mounted on standard aluminum specimen mounts utilizing double-sided adhesive tape with the open neural tube facing up. Samples were coated with gold to a $15 \mathrm{~nm}$ thickness using an EMS1550TES sputter coater (Electron Microscopy Sciences, Hatfield, PA), and imaged using a JEOL JSM5310 Scanning Electron Microscope, (JEOL, Inc. Peabody, MA) equipped with an Orion image acquisition and handling system imagegrabber (Model p13315A, JEOL, Inc. Peabody, MA). For imaging primary cilia of the yolk sac mesothelium, dissected yolk sacs were flat-mounted with the mesothelial side facing up prior to fixation.

Immunogold labeling for ADAMTS9 in RPE-1 cells was done using the preembedding technique. RPE-1 cells were plated in two-chamber Permanox slides (Lab-Tek, catalog no. 177429) and cultured to confluency before serum starvation for $48 \mathrm{~h}$. Cells were washed in PBS and fixed in 4\% PFA in PBST for $20 \mathrm{~min}$. Cells were washed and permeabilized with PBST for 30 min before blocking in 5\% normal goat serum for $1 \mathrm{~h}$ and overnight incubation with or without primary antibody against human ADAMTS9 (linker-2) at a 1:600 dilution at $4{ }^{\circ} \mathrm{C}$. Samples were washed in PBST for $30 \mathrm{~min}$ and incubated with blocking buffer $(0.1 \% \mathrm{BSA}$, $0.1 \%$ fish gelatin, and $0.2 \%$ Tween 20 in PBS) for an additional $30 \mathrm{~min}$. Goat antirabbit $5 \mathrm{~nm}$ gold-conjugated secondary antibody (EMS, catalog no. 15725) was diluted at 1:50 in blocking buffer and incubated for $4 \mathrm{~h}$ at room temperature. Samples were washed in blocking buffer for $30 \mathrm{~min}$ and fixed overnight in $2.5 \%$ glutaraldehyde, $4 \%$ PFA in $0.2 \mathrm{M}$ cacodylate buffer ( $\mathrm{pH}$ 7.3). Dehydrated samples were embedded in pure Eponate12 resin and polymerized overnight at room temperature. Eighty-five nanometers ultrathin sections were collected and stained with uranyl acetate and lead citrate and imaged using a FEI Tecnai G2 Spirit 

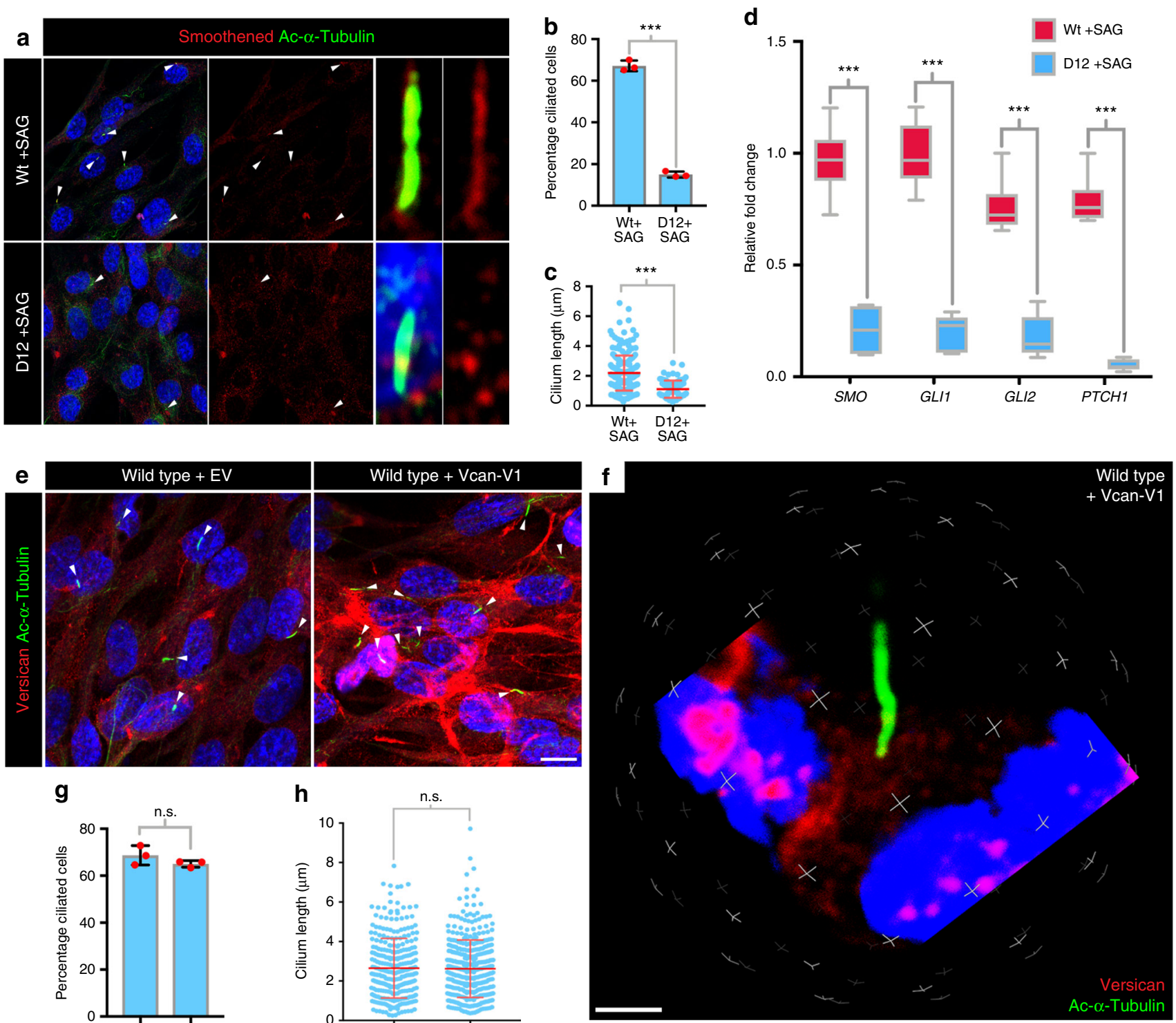
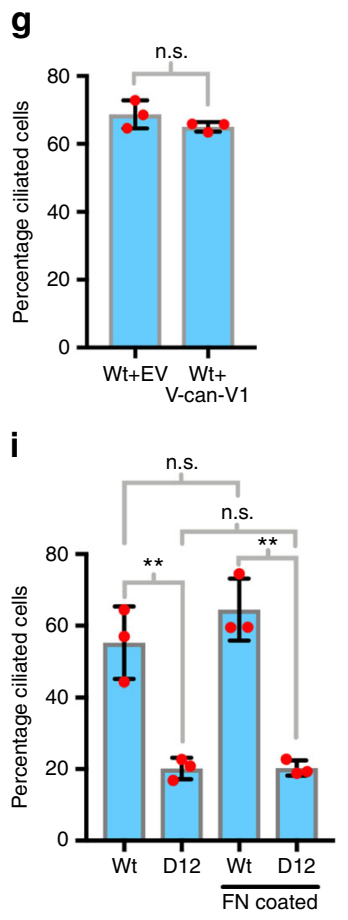
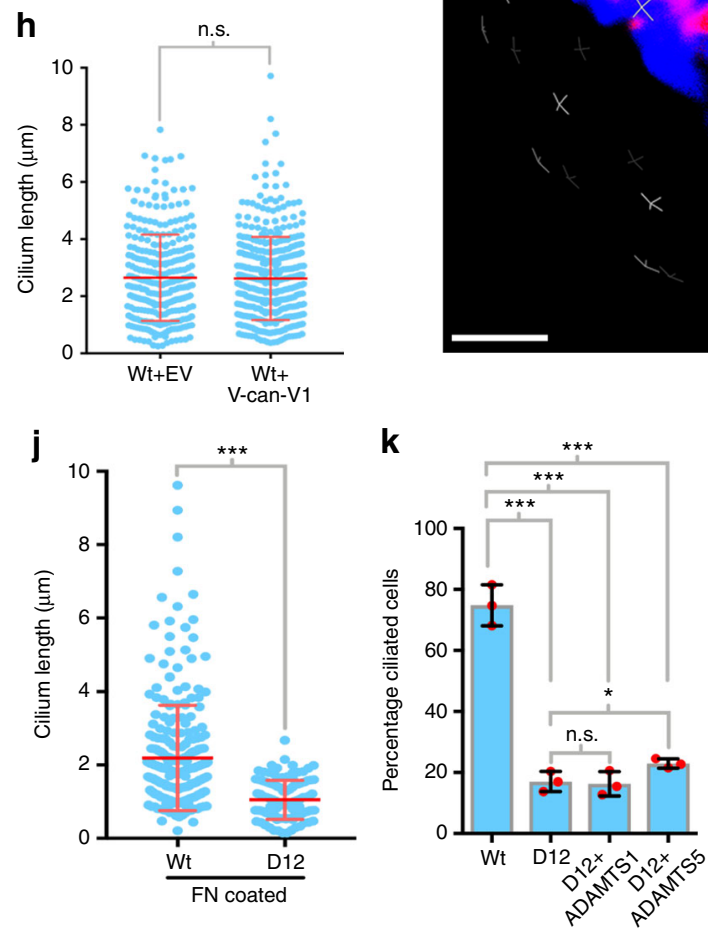

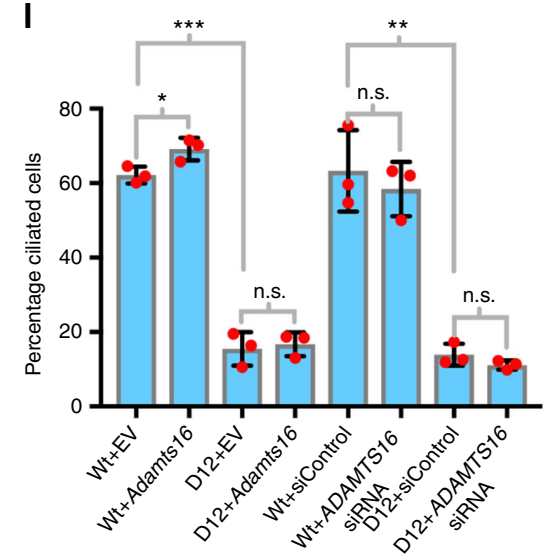

BioTWIN Transmission Electron Microscope (FEI company, Hillsboro, OR) equipped with an Orius 832 CCD camera (Gatan, Inc., Pleasanton, CA).

RNAscope in-situ hybridization. E8.5-E11.5 mouse embryos were fixed in 4\% PFA prior to paraffin embedding. Seven micrometer-thick sections were collected immediately prior to in-situ hybridization experiments using RNAscope probes specific for mouse Adamts9 (ACD, Cat. No. 400401), Adamts20 (ACD, Cat. No. 400541), Vcan exon 7 (GAG- $a$ domain) (ACD, Cat. No. 428311), Vcan exon 8 (GAG- $\beta$ domain) (ACD, Cat. No. 428321), Shh (ACD, Cat. No. 314361),

Ptch1 (ACD, Cat. No. 402811), Gli-1 (ACD, Cat. No. 311001), Gli-2 (ACD, Cat. No. 405771), Bmp4 (ACD, Cat. No. 401301), Noggin (ACD. Cat. No. 467391). 
Fig. 9 Cilium defects in ADAMTS9-null cells are independent of Shh signaling and versican cleavage. a Smoothened (red) and acetylated $\alpha$-tubulin (green) coimmunostaining of serum-starved wild-type RPE-1 and D12 cells treated with $500 \mathrm{~nm}$ SAG shows cilium-localized SMO in wild-type but not mutant cells. Arrowheads indicate primary cilia. b Percentage of ciliated cells in wild-type RPE-1 and D12 cells treated with $500 \mathrm{~nm}$ SAG ( $N=3$ independent experiments, ${ }^{\star \star \star} p<0.0001$, Student's $t$ test). c Quantification of cilium length in wild-type and D12 cells after treatment with 500 nm SAG shows no rescue of length ( $N$ $=3$ independent experiments, ${ }^{\star \star \star} p<0.0001$, Student's $t$ test). d qRT-PCR of SMO, GLI1, GLI2 and PTCH1 after 500 nm SAG treatment of wild-type and D12 cells shows attenuated response in mutant cells $\left(N=3\right.$ independent experiments, ${ }^{\star \star \star} p<0.0001$, Student's $t$ test). e, $\mathbf{f}$ Coimmunostaining of versican (red) and acetylated $\alpha$-tubulin (green) in wild-type cells transfected with empty vector or VCAN-V1 shows increased versican around wild-type cells without an effect on ciliation (arrowheads). $\mathbf{g}$ Percentage of cells with primary cilia in wild-type cells transfected with empty vector or VCAN-V1 (N $=3$ independent experiments, Student's $t$ test). $\mathbf{h}$ Quantification of primary cilium length in wild-type cells transfected with empty vector or VCAN-V1 ( $N=3$ independent experiments, Student's $t$ test). i Quantification of percentage of ciliated cells in wild-type and D12 cultures on $20 \mu \mathrm{g} / \mathrm{mL}$ fibronectin-coated or noncoated culture slides has no effect on wild-type or D12 cells ( $N=3$ independent experiments, ${ }^{\star \star} p<0.01$, Student's $t$ test). $\mathbf{j}$ Quantification of primary cilium length in wild-type and D12 cells cultured on $20 \mu \mathrm{g} / \mathrm{mL}$ fibronectin-coated slides did not restore D12 cilium length $\left(N=3\right.$ independent experiments, ${ }^{\star \star *} p<0.0001$, Student's $t$ test). $\mathbf{k}$ Transfection of ADAMTS1 or ADAMTS5 failed to rescue ciliogenesis in D12 cells ( $N=3$ experiments, ${ }^{\star \star \star} p<0.0001 ;{ }^{*} p<0.05$, Student's $t$ test). I Overexpression of Adamts16 or ADAMTS16 depletion failed to rescue ciliogenesis in D12 cells $\left(N=3\right.$ independent experiments, ${ }^{\star \star *} p<0.00001$; ${ }^{\star \star} p<0.001 ;{ }^{\star} p<0.05$, Student's $t$ test). Dotplot shows mean and SD. In box and whisker plot, whiskers indicate the range and boxes mark upper and lower quartiles. The centerline indicates the mean

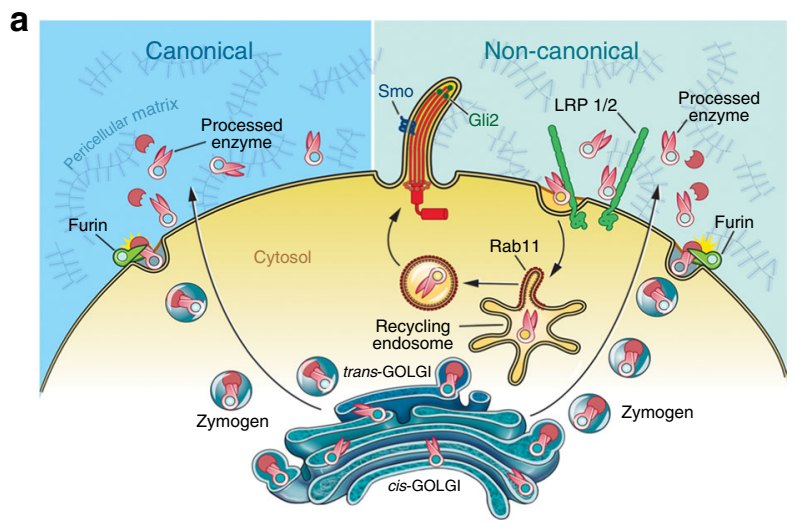

b

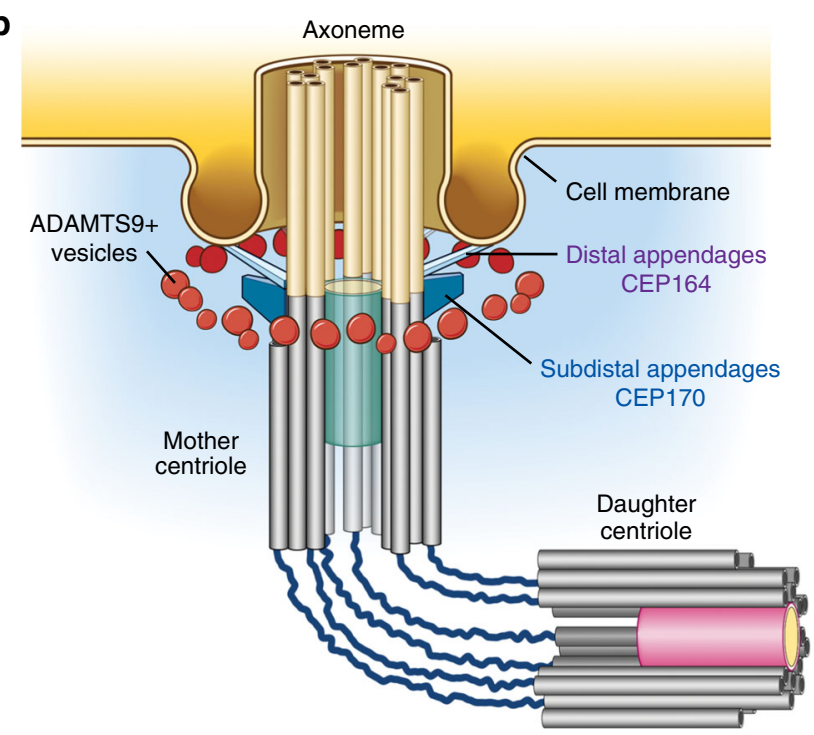

Fig. 10 The voyage of ADAMTS9 to the base of the primary cilium. a Schematic summarizing the life cycle and dual role of ADAMTS9 in ECM remodeling (left-hand side) and ciliogenesis (right-hand side). b Schematic illustrating the geographical distribution of ADAMTS9 vesicles at the ciliary base

Hybridization was done using a HybEZ oven and detected using the 2.5 HD Red detection kit. Hematoxylin was used as the counterstain. Sections were photographed on an Olympus BX51 upright microscope (Olympus, Center Valley, PA) using a Leica DFC7000T camera and Leica Application Suite v4.6 imaging software (both from Leica, Wetzlar, Germany).
Transferrin uptake assay. hTERT-RPE-1 cells grown to confluence in eightchamber cell culture slides and serum-starved for $24 \mathrm{~h}$ were cooled on ice for $10 \mathrm{~min}$ and the culture medium was switched to ice-cold serum-free medium for 5 min to block endocytosis. Alexa Fluor647-conjugated transferrin (Life Technologies, catalog no. T2366) was diluted in serum-free culture medium to a final concentration of $0.25 \mathrm{mg} / \mathrm{mL}$ and was prewarmed to $37^{\circ} \mathrm{C}$ before adding to the cells. Cells were incubated with medium containing Alexa Fluor647-transferrin at $37^{\circ} \mathrm{C}$ for $30 \mathrm{~min}$, washed thrice with serum-free medium, fixed in $4 \%$ PFA for $15 \mathrm{~min}$, washed and costained for ADAMTS 9 and acetylated $\alpha$-tubulin and imaged using super-resolution confocal microscopy at $\times 1000$ magnification.

Inhibition of endocytosis. hTERT-RPE-1 cells were grown to confluence in fourchamber cell culture slides and cultured in serum-free medium for $24 \mathrm{~h}$ to induce ciliogenesis. Dyngo4A (Abcam, catalog no. ab120689) and Pitstop 2 (Abcam, catalog no. ab120687) were dissolved in DMSO as $100 \mathrm{mM}$ stock solutions. Serumfree culture medium containing $20 \mu \mathrm{M}$ Dyngo4a or $10 \mu \mathrm{M}$ Pitstop-2 was prewarmed to $37^{\circ} \mathrm{C}$ and added to RPE-1 cells for $4-8 \mathrm{~h}$. Serum-free culture medium containing $0.2 \% \mathrm{DMSO}$ was used as a vehicle control. After incubation, cells were washed thrice in fresh serum-free culture medium, fixed in 4\% PFA diluted in PBS containing $0.1 \%$ Tween-20 (PBST), for $20 \mathrm{~min}$ and stained for acetylated $\alpha$-tubulin and ADAMTS9.

hTERT-RPE-1 cells grown to $80 \%$ confluence in four-chamber culture slides were transfected with Silencer ${ }^{\circledast}$ Select predesigned and validated siRNA targeting human CHC (s477, Ambion catalog no. 4390824), LRP-1 (106762, Ambion catalog no. AM51331) and LRP-2 (143880, Ambion catalog no. AM16708), or Silencer select negative control siRNA-2 (Ambion catalog no. 4390846) using Lipofectamine RNAiMAX reagent (Invitrogen, catalog no. 13778075). Cells were incubated with the transfection mix for $48 \mathrm{~h}$ for complete depletion of the target protein and washed in PBS and cultured for additional $24 \mathrm{~h}$ in serum-free medium for ciliogenesis. Treated cells were fixed in $4 \%$ paraformaldehyde in PBS containing $0.1 \%$ Tween 20 (PBST) for $20 \mathrm{~min}$ and stained for acetylated $\alpha$-tubulin and ADAMTS9.

SAG treatment of RPE-1 cells. Wild-type or D12 hTERT-RPE-1 cells were grown to confluence in eight-chamber cell culture slides for immunostaining, or six-well culture plates for RNA extraction, and cultured in serum-free medium for $24 \mathrm{~h}$ to induce ciliogenesis. Serum-free culture medium containing $500 \mathrm{nM}$ InSolution Smoothened Agonist (SAG) (EMD Milipore, Cat. \# 566661) was added to cells for $12 \mathrm{~h}$. Cells cultured in chamber slides were washed in PBS and fixed in 4\% PFA for analysis by immunostaining and cells cultured in six-well plates were washed in PBS and RNA was extracted using TRIzol RNA extraction reagent (Thermo Fisher, Cat. \# 15596026).

Isolation of HS and HS coupling to HiTrap columns for FPLC. HS columns were generated as follows: $1 \mathrm{~g}$ (wet weight) C57/BL6 mouse embryos (E12-E18) were homogenized and digested in $320 \mathrm{mM} \mathrm{NaCl}, 100 \mathrm{mM}$ sodium acetate (pH 5.5) containing $1 \mathrm{mg} / \mathrm{mL}$ pronase, and $1 \mathrm{mg} / \mathrm{mL}$ proteinase $\mathrm{K}$ for $72 \mathrm{~h}$ at $40^{\circ} \mathrm{C}$. Fresh enzymes were added every $12 \mathrm{~h}$. The digested samples were centrifuged, filtered, diluted 1:3 in water and $2.5 \mathrm{~mL}$ aliquots were applied to DEAE Sephacel columns. Eluted glycosaminoglycans were lyophilized, diluted, and quantified by the carbazole reaction. The material was then coupled to NHS-activated sepharose columns. The columns were tested using recombinant HS-binding proteins fibroblast growth factors 2 and 8 , vascular endothelial growth factor and Semaphorin $3 \mathrm{~F}$ as positive controls. FPLC was conducted on an Äkta protein purifier (GE Healthcare) at $8{ }^{\circ} \mathrm{C}$. Samples were applied to the columns in the absence of salt, and bound 
material was eluted by using a linear 0 to $1.5 \mathrm{M} \mathrm{NaCl}$ gradient in $0.1 \mathrm{M}$ phosphate buffer ( $\mathrm{pH}$ 7.0). Eluted fractions were TCA precipitated and analyzed by SDSPAGE. Signals were quantified by using ImageJ. For heparin affinity chromatography, commercial heparin Sepharose columns (GE Healthcare) were used.

Western blotting. SDS-PAGE was carried out on 7.5\% acrylamide gels or 4-20\% gradient gels under reducing conditions. Proteins were transferred to ImmobilonFL transfer membranes (EMD Milipore, cat. no. IPFL00010) and blocked using 5\% nonfat dry milk made in PBST. Primary antibodies were diluted in blocking buffer and incubated overnight at $4{ }^{\circ} \mathrm{C}$. LI-COR IR dye secondary antibodies (LI-COR Biosciences, Lincoln, NE), goat anti-mouse IR dye 800CW (Catalog no. 92632210), or goat anti-rabbit IR dye 680RD (Catalog no. 926-68071) were used at $1: 10,000$ dilutions in PBS to detect primary antibodies. Uncropped images of all blots can be found in Supplementary Fig. 8.

Western blot band intensity quantifications. A LI-COR Odyssey CLx scanner (LI-COR Biosciences, Lincoln, NE) operated using LI-COR Image Studio (ver. 4.0) was used to scan antibody-stained western blot membranes and for quantification of band intensities. Experimental band intensities were normalized to GAPDH intensity measured in each lane. Statistical significance of normalized band intensities was analyzed and graphed using the GraphPad Prism software (La Jolla, CA). Statistical significance was determined by an unpaired two-tailed Student's $t$ test.

Fluorescent microscopy image quantifications. For quantifying vesicle diameters and distances, super-resolution confocal microscopy images obtained at $\times 1000$ optical magnification were measured by the Leica LAS X software (version 3.1.5.16308) distance measurement function as shown in Fig. 1j. For quantifying ADAMTS9 pixel intensities at the base of the cilium, conventional confocal microscopy images (nondeconvoluted) were analyzed by ImageJ. In brief, a boxed region of interest (ROI) was drawn directly underneath the axoneme and transferred to the corresponding ADAMTS9 channel (red/568 channel), and the arbitrary mean pixel intensity for the ROI was measured. The "restore selection" function was used to transfer the same ROI to all images analyzed. To determine primary cilium length in cultured cells, the freehand distance measurement tool in Image $(\mathrm{NIH})$ was used to measure the acetylated $\alpha$-tubulin staining signal of fluorescent images taken at $\times 100$ magnification. To determine primary cilium length in mouse neural tubes and yolk sacs the freehand distance measurement tool in ImageJ was used to trace the primary cilium in SEM images. The GraphPad Prism software (La Jolla, CA) was used to determine statistical significance using an unpaired two-tailed Student's $t$ test.

\section{Data availability}

The data that support the findings of this study are available from the Lead Contact, Dr. Suneel Apte (aptes@ccf.org). Requests for resources and reagents should also be directed to the Lead Contact, Dr. Suneel Apte (aptes@ccf.org).

Received: 21 May 2018 Accepted: 11 January 2019

Published online: 27 February 2019

\section{References}

1. Marchant, D. J. et al. A new transcriptional role for matrix metalloproteinase12 in antiviral immunity. Nat. Med. 20, 493-502 (2014).

2. Shimizu-Hirota, R. et al. MT1-MMP regulates the PI3Kdelta.Mi-2/NuRDdependent control of macrophage immune function. Genes Dev. 26, 395-413 (2012).

3. Bangs, F. K., Schrode, N., Hadjantonakis, A. K. \& Anderson, K. V. Lineage specificity of primary cilia in the mouse embryo. Nat. Cell Biol. 17, 113-122 (2015).

4. Mick, D. U. et al. Proteomics of primary cilia by proximity labeling. Dev Cell. 35, 497-512 (2015).

5. Sasai, N. \& Briscoe, J. Primary cilia and graded Sonic Hedgehog signaling. Wiley Interdiscip. Rev. Dev. Biol. 1, 753-772 (2012).

6. Reiter, J. F., Blacque, O. E. \& Leroux, M. R. The base of the cilium: roles for transition fibres and the transition zone in ciliary formation, maintenance and compartmentalization. EMBO Rep. 13, 608-618 (2012).

7. Kim, J. et al. Functional genomic screen for modulators of ciliogenesis and cilium length. Nature 464, 1048-1051 (2010).

8. Milenkovic, L., Scott, M. P. \& Rohatgi, R. Lateral transport of Smoothened from the plasma membrane to the membrane of the cilium. J. Cell. Biol. 187, 365-374 (2009).

9. Reiter, J. F. \& Leroux, M. R. Genes and molecular pathways underpinning ciliopathies. Nat. Rev. Mol. Cell Biol. 18, 533-547 (2017).
10. Clement, C. A. et al. TGF-beta signaling is associated with endocytosis at the pocket region of the primary cilium. Cell Rep. 3, 1806-1814 (2013).

11. Blelloch, R. \& Kimble, J. Control of organ shape by a secreted metalloprotease in the nematode Caenorhabditis elegans. Nature 399, 586-590 (1999).

12. Somerville, R. P. et al. Characterization of ADAMTS-9 and ADAMTS-20 as a distinct ADAMTS subfamily related to Caenorhabditis elegans GON-1. J. Biol. Chem. 278, 9503-9513 (2003).

13. Rao, C. et al. A defect in a novel ADAMTS family member is the cause of the belted white-spotting mutation. Development 130, 4665-4672 (2003).

14. Silver, D. L. et al. The secreted metalloprotease ADAMTS20 is required for melanoblast survival. PLoS Genet. 4, 1-15 (2008).

15. Wolf, Z. T. et al. Genome-wide association studies in dogs and humans identify ADAMTS20 as a risk variant for cleft lip and palate. PLoS Genet. 11, e1005059 (2015).

16. Enomoto, H. et al. Cooperation of two ADAMTS metalloproteases in closure of the mouse palate identifies a requirement for versican proteolysis in regulating palatal mesenchyme proliferation. Development 137, 4029-4038 (2010).

17. Nandadasa, S., Nelson, C. M. \& Apte, S. S. ADAMTS9-mediated extracellular matrix dynamics regulates umbilical cord vascular smooth muscle differentiation and rotation. Cell Rep. 11, 1519-1528 (2015).

18. McCulloch, D. R. et al. ADAMTS metalloproteases generate active versican fragments that regulate interdigital web regression. Dev. Cell 17, 687-698 (2009).

19. Graser, S. et al. Cep164, a novel centriole appendage protein required for primary cilium formation. J. Cell Biol. 179, 321-330 (2007).

20. Koo, B. H. et al. Cell-surface processing of pro-ADAMTS9 by furin. J. Biol. Chem. 281, 12485-12494 (2006).

21. Molla-Herman, A. et al. The ciliary pocket: an endocytic membrane domain at the base of primary and motile cilia. J. Cell Sci. 123, 1785-1795 (2010).

22. Zou, K. et al. A heparin-binding growth factor, midkine, binds to a chondroitin sulfate proteoglycan, PG-M/versican. Eur. J. Biochem. 267, 4046-4053 (2000)

23. Wang, L. \& Dynlacht, B. D. The regulation of cilium assembly and disassembly in development and disease. Development 145, dev151407 (2018).

24. Nachury, M. V. et al. A core complex of BBS proteins cooperates with the GTPase Rab8 to promote ciliary membrane biogenesis. Cell 129, 1201-1213 (2007).

25. Westlake, C. J. et al. Primary cilia membrane assembly is initiated by Rab11 and transport protein particle II (TRAPPII) complex-dependent trafficking of Rabin8 to the centrosome. Proc. Natl. Acad. Sci. USA 108, 2759-2764 (2011).

26. Yee, L. E. \& Reiter, J. F. Ciliary vesicle formation: a prelude to ciliogenesis. Dev. Cell 32, 665-666 (2015).

27. Yamamoto, K. et al. Low density lipoprotein receptor-related protein 1 (LRP1)-mediated endocytic clearance of a disintegrin and metalloproteinase with thrombospondin motifs-4 (ADAMTS-4): functional differences of noncatalytic domains of ADAMTS-4 and ADAMTS-5 in LRP1 binding. J. Biol. Chem. 289, 6462-6474 (2014).

28. Christ, A. et al. LRP2 acts as SHH clearance receptor to protect the retinal margin from mitogenic stimuli. Dev. Cell 35, 36-48 (2015).

29. Kashiwagi, M., Tortorella, M., Nagase, H. \& Brew, K. TIMP-3 is a potent inhibitor of aggrecanase 1 (ADAM-TS4) and aggrecanase 2 (ADAM-TS5). J. Biol. Chem. 276, 12501-12504 (2001).

30. Kobayashi, T., Tsang, W. Y., Li, J., Lane, W. \& Dynlacht, B. D. Centriolar kinesin Kif24 interacts with CP110 to remodel microtubules and regulate ciliogenesis. Cell 145, 914-925 (2011).

31. Cao, J. et al. miR-129-3p controls cilia assembly by regulating CP110 and actin dynamics. Nat. Cell Biol. 14, 697-706 (2012).

32. Lu, Q. et al. Early steps in primary cilium assembly require EHD1/EHD3dependent ciliary vesicle formation. Nat. Cell Biol. 17, 228-240 (2015).

33. Sanchez, I. \& Dynlacht, B. D. Cilium assembly and disassembly. Nat. Cell Biol. 18, 711-717 (2016).

34. Mjaatvedt, C. H., Yamamura, H., Capehart, A. A., Turner, D. \& Markwald, R. $\mathrm{R}$. The Cspg2 gene, disrupted in the hdf mutant, is required for right cardiac chamber and endocardial cushion formation. Dev. Biol. 202, 56-66 (1998).

35. Haycraft, C. J. et al. Gli2 and Gli3 localize to cilia and require the intraflagellar transport protein polaris for processing and function. PLoS Genet. 1, e53 (2005).

36. Hoover, A. N. et al. C2cd3 is required for cilia formation and Hedgehog signaling in mouse. Development 135, 4049-4058 (2008).

37. Huangfu, D. et al. Hedgehog signalling in the mouse requires intraflagellar transport proteins. Nature 426, 83-87 (2003).

38. Liu, A., Wang, B. \& Niswander, L. A. Mouse intraflagellar transport proteins regulate both the activator and repressor functions of Gli transcription factors. Development 132, 3103-3111 (2005).

39. Briscoe, J. \& Therond, P. P. The mechanisms of Hedgehog signalling and its roles in development and disease. Nat. Rev. Mol. Cell Biol. 14, 416-429 (2013). 
40. Dessaud, E., McMahon, A. P. \& Briscoe, J. Pattern formation in the vertebrate neural tube: a sonic hedgehog morphogen-regulated transcriptional network. Development 135, 2489-2503 (2008).

41. Timmer, J. R., Wang, C. \& Niswander, L. BMP signaling patterns the dorsal and intermediate neural tube via regulation of homeobox and helix-loop-helix transcription factors. Development 129, 2459-2472 (2002).

42. Veleri, S. et al. Ciliopathy-associated gene Cc2d2a promotes assembly of subdistal appendages on the mother centriole during cilia biogenesis. Nat. Commun. 5, 4207 (2014)

43. Sang, L. et al. Mapping the NPHP-JBTS-MKS protein network reveals ciliopathy disease genes and pathways. Cell 145, 513-528 (2011).

44. Goodrich, L. V., Milenkovic, L., Higgins, K. M. \& Scott, M. P. Altered neural cell fates and medulloblastoma in mouse patched mutants. Science 277, 1109-1113 (1997).

45. Dubail, J. et al. A new Adamts9 conditional mouse allele identifies its nonredundant role in interdigital web regression. Genesis 52, 702-712 (2014).

46. Jungers, K. A., Le Goff, C., Somerville, R. P. \& Apte, S. S. Adamts9 is widely expressed during mouse embryo development. Gene Expr. Patterns 5, 609-617 (2005).

47. Dours-Zimmermann, M. T. \& Zimmermann, D. R. A novel glycosaminoglycan attachment domain identified in two alternative splice variants of human versican. J. Biol. Chem. 269, 32992-32998 (1994).

48. Zimmermann, D. R. \& Ruoslahti, E. Multiple domains of the large fibroblast proteoglycan, versican. EMBO J. 8, 2975-2981 (1989).

49. Sandy, J. D. et al. Versican V1 proteolysis in human aorta in vivo occurs at the Glu441- Ala442 bond, a site that is cleaved by recombinant ADAMTS-1 and ADAMTS- 4. J. Biol. Chem. 276, 13372-13378 (2001).

50. Yamagata, M., Yamada, K. M., Yoneda, M., Suzuki, S. \& Kimata, K. Chondroitin sulfate proteoglycan (PG-M-like proteoglycan) is involved in the binding of hyaluronic acid to cellular fibronectin. J. Biol. Chem. 261, 13526-13535 (1986).

51. Liu, J. et al. Sonic hedgehog signaling directly targets Hyaluronic Acid Synthase 2, an essential regulator of phalangeal joint patterning. Dev. Biol. 375, 160-171 (2013).

52. Byrd, N. et al. Hedgehog is required for murine yolk sac angiogenesis. Development 129, 361-372 (2002).

53. Gopalakrishnan, K. et al. Targeted disruption of Adamts16 gene in a rat genetic model of hypertension. Proc. Natl. Acad. Sci. USA 109, 20555-20559 (2012).

54. Choi, Y. J. et al. Mutations of ADAMTS9 cause nephronophthisis-related ciliopathy.Am.J.Hum.Genet. 104, 45-54 (2019).

55. Benz, B. A., et al. Genetic and biochemical evidence that gastrulation defects in Pofut2 mutants result from defects in ADAMTS9 secretion. Dev. Biol. 416, 111-122 (2016).

56. Dubail, J. et al. Impaired ADAMTS9 secretion: a potential mechanism for eye defects in Peters Plus Syndrome. Sci. Rep. 6, 33974 (2016).

57. Koo, B. H. \& Apte, S. S. Cell-surface processing of the metalloprotease proADAMTS9 is influenced by the chaperone GRP94/gp96. J. Biol. Chem. 285 , 197-205 (2010).

58. Koo, B. H. et al. Regulation of ADAMTS9 secretion and enzymatic activity by its propeptide. J. Biol. Chem. 282, 16146-16154 (2007).

59. Sorokin, S. Centrioles and the formation of rudimentary cilia by fibroblasts and smooth muscle cells. J. Cell Biol. 15, 363-377 (1962).

60. Doyotte, A., Mironov, A., McKenzie, E. \& Woodman, P. The Brol-related protein HD-PTP/PTPN23 is required for endosomal cargo sorting and multivesicular body morphogenesis. Proc. Natl. Acad. Sci. USA 105, 6308-6313 (2008).

61. Inoue, H., Ha, V. L., Prekeris, R. \& Randazzo, P. A. Arf GTPase-activating protein ASAP1 interacts with Rab11 effector FIP3 and regulates pericentrosomal localization of transferrin receptor-positive recycling endosome. Mol. Biol. Cell 19, 4224-4237 (2008).

62. Franco, I. et al. PI3K class II alpha controls spatially restricted endosomal PtdIns3P and Rab11 activation to promote primary cilium function. Dev. Cell 28, 647-658 (2014).

63. Gao, J. et al. Laminin-511 is an epithelial message promoting dermal papilla development and function during early hair morphogenesis. Genes Dev. 22, 2111-2124 (2008).

64. Hsiao, Y. C., Tuz, K. \& Ferland, R. J. Trafficking in and to the primary cilium. Cilia 1, 4 (2012).

65. Bandari, S. et al. Sweet on Hedgehogs: regulatory roles of heparan sulfate proteoglycans in Hedgehog-dependent cell proliferation and differentiation. Curr. Protein Pept. Sci. 16, 66-76 (2015).

66. Ortmann, C. et al. Sonic hedgehog processing and release are regulated by glypican heparan sulfate proteoglycans. J. Cell. Sci. 128, 2374-2385 (2015).

67. Nikolopoulou, E., Galea, G. L., Rolo, A., Greene, N. D. \& Copp, A. J. Neural tube closure: cellular, molecular and biomechanical mechanisms. Development 144, 552-566 (2017).
68. McGlashan, S. R., Jensen, C. G. \& Poole, C. A. Localization of extracellular matrix receptors on the chondrocyte primary cilium. J. Histochem. Cytochem. 54, 1005-1014 (2006)

69. Mead, T. J. et al. ADAMTS9-regulated pericellular matrix dynamics Governs focal adhesion-dependent smooth muscle differentiation. Cell Rep. $\mathbf{2 3}$ 485-498 (2018).

70. Yamamura, H., Zhang, M., Markwald, R. R. \& Mjaatvedt, C. H. A heart segmental defect in the anterior-posterior axis of a transgenic mutant mouse. Dev. Biol. 186, 58-72 (1997).

71. Apte, S. S., Olsen, B. R. \& Murphy, G. The gene structure of tissue inhibitor of metalloproteinases (TIMP)-3 and its inhibitory activities define the distinct TIMP gene family. J. Biol. Chem. 270, 14313-14318 (1995).

72. Longpre, J. M. \& Leduc, R. Identification of prodomain determinants involved in ADAMTS-1 biosynthesis. J. Biol. Chem. 279, 33237-33245 (2004).

73. Longpre, J. M. et al. Characterization of proADAMTS5 processing by proprotein convertases. Int. J. Biochem. Cell Biol. 41, 1116-1126 (2009).

74. Schnellmann, R. et al. A selective extracellular matrix proteomics approach identifies fibronectin proteolysis by ADAMTS16 and its impact on spheroid morphogenesis. Mol. Cell. Proteom. https://doi.org/10.1074/mcp.RA118.000676 (2018).

75. Dutt, S. et al. Versican V0 and V1 direct the growth of peripheral axons in the developing chick hindlimb. J. Neurosci. 31, 5262-5270 (2011).

\section{Acknowledgements}

Funding was provided by the NIH-NHLBI Program of Excellence in Glycosciences award HL107147 and NIH-NEI award EY024943 to S.S.A. and the David and Lindsay Morgenthaler Postdoctoral Fellowship and the Mark Lauer Pediatric Research Grant to S. N. This research was supported by the Allen Distinguished Investigator Program, through support made by The Paul G. Allen Frontiers Group and the American Heart Association to S.S.A. This work utilized a Leica SP8 confocal microscope that was purchased with funding from National Institutes of Health SIG grant 1S10OD019972-01 We thank Apte laboratory members and Dr. Jeremy Reiter for valuable discussions, Johanne Dubail and Rahel Schnellmann for ADAMTS9 and ADAMTS16 plasmids, Judy Drazba and Mei Yin of the Lerner Research Institute Imaging Core for guidance with microscopy, and Dave Schumick of the LRI Medical Art and Photography department for Figs. 4l, m, 10 .

\section{Author contributions}

S.S.A., S.N., H.Y.G., and F.H. conceived and designed the experiments. S.N., L.W.W., A.O., C.M.K, R.P., K.G., T.C.C., and H.Y.G. performed the experiments. S.S.A. and S.N. wrote the manuscript. All authors read and approved the manuscript.

\section{Additional information}

Supplementary Information accompanies this paper at https://doi.org/10.1038/s41467 019-08520-7.

Competing interests: The authors declare no competing interests.

Reprints and permission information is available online at http://npg.nature.com/ reprintsandpermissions/

Journal peer review information: Nature Communications thanks Benjamin Allen and the other anonymous reviewers for their contribution to the peer review of this work. Peer reviewer reports are available.

Publisher's note: Springer Nature remains neutral with regard to jurisdictional claims in published maps and institutional affiliations.

Open Access This article is licensed under a Creative Commons Attribution 4.0 International License, which permits use, sharing, adaptation, distribution and reproduction in any medium or format, as long as you give appropriate credit to the original author(s) and the source, provide a link to the Creative Commons license, and indicate if changes were made. The images or other third party material in this article are included in the article's Creative Commons license, unless indicated otherwise in a credit line to the material. If material is not included in the article's Creative Commons license and your intended use is not permitted by statutory regulation or exceeds the permitted use, you will need to obtain permission directly from the copyright holder. To view a copy of this license, visit http://creativecommons.org/ licenses/by/4.0/

(C) The Author(s) 2019 